\title{
The Spectra of Certain Classes of Room Frames: The Last Cases
}

\author{
Jeffrey H. Dinitz and Gregory S. Warrington \\ Department of Mathematics and Statistics \\ University of Vermont \\ Burlington, Vermont \\ U.S.A. 05405
}

Submitted: Nov 13, 2009; Accepted: May 5, 2010; Published: May 20, 2010

Mathematics Subject Classification: 05B15

\begin{abstract}
In this paper we study the spectra of certain classes of Room frames. The three spectra which we study are incomplete Room squares, uniform Room frames and Room frames of type $2^{u} t^{1}$. These problems have been studied in numerous papers over the years; in this paper, we complete the three spectra. In addition we find a Howell cube of type $H_{3}(6,10)$. This corrects a previous claim of nonexistence of this design.
\end{abstract}

\section{Introduction}

Room squares and generalizations have been extensively studied for over 40 years. In 1974, Mullin and Wallis [12] showed that the spectrum of Room squares consists of all odd positive integers other than 3 or 5; however, many other related questions have remained unsolved. (For an extensive survey from 1992 of Room squares and related designs, we refer the reader to [5].) In the 1994 paper by Dinitz, Stinson and Zhu [6], the authors studied three well-known spectra of designs closely related to Room squares and in each instance left exactly one unsolved case. In this paper we will prove the existence of each of these designs.

Also, in the 1986 paper by A. Rosa and D. Stinson [13] it was claimed that there is no Howell cube on any 6-regular graph on 10 points. In Section 2 we disprove this claim by exhibiting such a cube.

We begin with the definitions. Let $S$ be a set, and let $\left\{S_{1}, \ldots, S_{n}\right\}$ be a partition of $S$. An $\left\{S_{1}, \ldots, S_{n}\right\}$-Room frame is an $|S| \times|S|$ array, $F$, indexed by $S$, which satisfies the following properties: 
1. every cell of $F$ either is empty or contains an unordered pair of symbols of $S$,

2. the subarrays $S_{i} \times S_{i}$ are empty, for $1 \leqslant i \leqslant n$ (these subarrays are referred to as holes),

3. each symbol $x \notin S_{i}$ occurs once in row (or column) $s$, for any $s \in S_{i}$,

4. the pairs occurring in $F$ are those $\{s, t\}$, where $(s, t) \in(S \times S) \backslash \cup_{i=1}^{n}\left(S_{i} \times S_{i}\right)$.

The type of a Room frame $F$ is defined to be the multiset $\left\{\left|S_{i}\right|: 1 \leqslant i \leqslant n\right\}$. Typically an "exponential" notation is used to describe types: type $t_{1}{ }^{u_{1}} t_{2}{ }^{u_{2}} \cdots t_{k}{ }^{u_{k}}$ denotes $u_{i}$ occurrences of $t_{i}, 1 \leqslant i \leqslant k$. We note that a Room square of side $n$ is equivalent to a Room frame of type $1^{n}$. Examples of Room frames of types $1^{8} 3^{1}$ and $2^{5}$ are given below.

\begin{tabular}{|c|c|c|c|c|c|c|c|c|c|c|}
\hline \multicolumn{2}{|c|}{} & 48 & & & 37 & $6 X$ & & & 59 \\
\cline { 4 - 11 } \multicolumn{2}{|c|}{} & & 69 & & & $5 X$ & 38 & & & 47 \\
\hline & & 39 & $4 X$ & & & 57 & 68 & \\
\hline 67 & $8 X$ & & & & & 04 & 15 & 29 & & \\
\hline 58 & 79 & & & & 03 & & & & $2 X$ & 16 \\
\hline $9 X$ & & 78 & & 06 & & & 24 & & 13 & \\
\hline & & & 05 & $7 X$ & 89 & & & 14 & & 23 \\
\hline & 46 & $3 X$ & & 25 & & 19 & & & & 08 \\
\hline & 35 & 49 & $1 X$ & & 26 & & & & 07 & \\
\hline 34 & & 56 & & & 17 & 28 & & $0 X$ & & \\
\hline & & & 27 & 18 & & & 09 & 36 & 45 & \\
\hline
\end{tabular}

A Room frame of type $1^{8} 3^{1}$

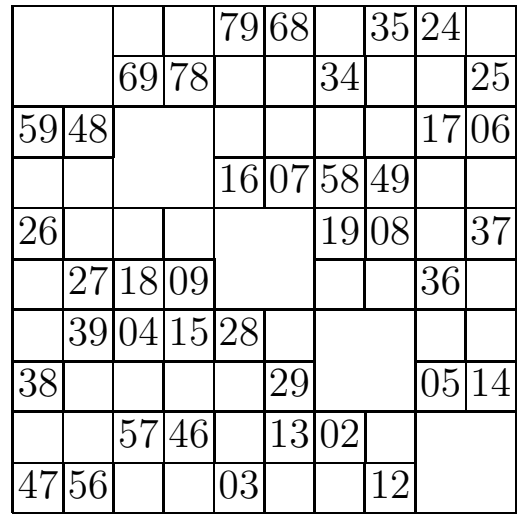

A (uniform) Room frame of type $2^{5}$

Note that the pairs of elements contained in a Room square naturally define a graph (the underlying graph). Each row (column) of the Room frame is a 1-factor of the underlying graph and the set of all rows (columns) is a 1-factorization of the underlying graph. The row 1-factorization and column 1-factorization are orthogonal 1-factorizations in the sense that any two edges that are in the same row 1-factor are in different column 1-factors. It is straightforward to see that the existence of a Room frame of type $t_{1}{ }^{u_{1}} t_{2}{ }^{u_{2}} \ldots t_{k}{ }^{u_{k}}$ is equivalent to a pair of orthogonal 1-factorizations of the complete graph on $\sum t_{i} \times u_{i}$ points which is missing a spanning set of $u_{i}$ complete graphs on $t_{i}$ points for $1 \leqslant i \leqslant k$.

Three important spectra of Room frames were considered in [6] and will be discussed in this paper. Specifically, they are Room frames of types $1^{n-s} s^{1}$ (incomplete Room squares), $t^{u}$ (uniform Room frames) and $2^{u} t^{1}$. We describe each one and give the current state of knowledge for each.

The existence of a Room frame of type $1^{n-s} s^{1}$ is equivalent to the existence of an object called an $(n, s)$-incomplete Room square which is essentially a Room square of side $n$ containing a Room square of side $s$ as a subarray. (By considering "incomplete" Room squares, one can allow $s=3$ or 5 , as well.) The existence of these objects is a fundamental question in this area. See $[5,6,7,14]$ for prior results. The following theorem summarizes the known results regarding these objects. 
Theorem 1.1 [6] Suppose $n$ and $s$ are odd positive integers, $n \geqslant 3 s+2$, and $(n, s) \neq$ $(5,1)$. Then there exists an $1^{n-s} s^{1}$ Room frame (equivalently an $(n, s)$-incomplete Room square) except possibly when $n=67$ and $s=21$.

A Room frame of type $t^{u}$ (i.e. a Room frame having $u$ holes, each of size $t$ ) is termed a uniform Room frame. A systematic study of the spectrum for uniform Room frames was begun in 1981 in [3]. Other results can be found in [2, 5, 6, 9]. The following theorem summarizes the known results regarding uniform Room frames.

Theorem 1.2 [6] Suppose $t$ and $u$ are positive integers, $u \geqslant 4$, and $(t, u) \neq(1,5),(2,4)$. Then there exists a Room frame of type $t^{u}$ if and only if $t(u-1)$ is even, except possibly when $u=4$ and $t=14$ (i.e. of type $14^{4}$ ).

Room frames of type $2^{u} t^{1}$ are Room frames with one hole of size $t$ and $u$ holes of size 2 . This problem can be thought of as an even-side analogue of the incomplete Room square problem. The known results on this problem can be found in $[6,8,10]$. The following theorem summarizes the known results regarding this type of frame.

Theorem 1.3 [6] Suppose $t$ and $u$ are positive integers. If $t \geqslant 4$, then there exists a frame of type $2^{u} t^{1}$ if and only if $t$ is even and $u \geqslant t+1$, except possibly when $u=19$ and $t=18$.

In this paper we find all three of the exceptional cases, allowing us to complete each of the spectra mentioned above. Our method entails the use of the hill-climbing algorithm for finding Room frames. This algorithm is described in [4] and was used previously to find many of the frames of the types mentioned in this paper. An additional description of the algorithm can be found at [5] and a general description of hill-climbing algorithms used in design theory can be found at [11]. We should mention that the current success of the algorithm is mainly attributable to the speedup of computers (and the existence of large clusters) over the past 15 years. There were no new heuristics or algorithmic speedups used in these searches over what was used in [6].

We ran the algorithm many times and below we give a statistical analysis of expected number times the algorithm must be restarted before finding each frame. It also estimates the expected time for a single process to finish on a single $3 \mathrm{GHz} c p u$. In each case a restart begins when the search reaches the threshold of $8000 *$ side operations without a decrease in the deficit. A rough estimate of the time this algorithm could have taken to complete these searches in 1994 would be to multiply the value given below by 40 .

\begin{tabular}{|c|c|c|}
\hline frame & number of restarts & expected time to finish (hours) \\
\hline $1^{46} 21^{1}$ & 480,000 & 16 \\
$2^{19} 18^{1}$ & 550,000 & 18 \\
$14^{4}$ & $5,260,000$ & 180 \\
\hline
\end{tabular}


In the Appendix we give a $(67,21)$-incomplete Room square, a Room frame of type $14^{4}$ and a Room frame of type $2^{19} 18^{1}$. We present each as a pair of orthogonal onefactorizations $F_{r}$ and $F_{c}$ of the appropriate underlying graph (for example $K_{68}-K_{22}$ for the $(67,21)$-incomplete Room square). In order to construct the square just note that if pair $\{x, y\}$ is in the $i$ th factor of $F_{r}$ and the $j$ th factor of $F_{c}$, then the pair $\{x, y\}$ is in the $i$ th row and $j$ th column of the Room frame. The addition of these frames to the three theorems above completes each of their spectra. We record this in the following three theorems.

Theorem 1.4 There exists an $1^{n-s} s^{1}$ Room frame (equivalently an $(n, s)$-incomplete Room square) if and only if $n$ and $s$ are odd positive integers, $n \geqslant 3 s+2$, and $(n, s) \neq$ $(5,1)$.

Theorem 1.5 There exists a Room frame of type $t^{u}$ if and only if $u \geqslant 4, t(u-1)$ is even, and $(t, u) \neq(1,5),(2,4)$.

Theorem 1.6 There exists a frame of type $2^{u} t^{1}$ if and only if $t$ and $u$ are positive integers, $t$ is even and $u \geqslant t+1$.

\section{A Howell cube}

An object that is very closely related to a Room square and slightly more general is a Howell design. Let $S$ be a set of $2 n$ symbols. A Howell design $H(s, 2 n)$ (on symbol set $S$ ) is an $s \times s$ array, $H$, which satisfies the properties:

1. every cell of $H$ either is empty or contains an unordered pair of symbols from $S$,

2. each symbol of $S$ occurs once in each row and column of $H$, and

3. every unordered pair of symbols occurs in at most one cell of $H$.

We note that a Room square of side $2 n-1$ is an $\mathrm{H}(2 n-1,2 n)$. As was the case for Room frames, the pairs of symbols in the cells of an $H(s, 2 n)$ can be thought of as the edges of a $s$ regular graph on $2 n$ symbols, the underlying graph of the Howell design. An $H^{*}(s, 2 n)$ is defined to be an $H(s, 2 n)$ whose underlying graph contains a maximal independent set, i.e. one of size $2 n-s$. The rows and columns of an $\mathrm{H}(s, 2 n)$ form orthogonal 1-factorizations of the underlying graph. As with Room frames, the existence of a pair of orthogonal 1-factorizations of an $s$-regular graph on $2 n$ vertices is equivalent to the existence of an $\mathrm{H}(s, 2 n)$. Below we give examples of two small Howell designs, an $\mathrm{H}(4,6)$ and an $\mathrm{H}^{*}(4,8)$ with independent set $\{1,2,3,4\}$.

\begin{tabular}{|l|l|l|l|}
\hline 04 & & 13 & 25 \\
\hline 23 & 14 & 05 & \\
\hline & 35 & 24 & 01 \\
\hline 15 & 02 & & 34 \\
\hline
\end{tabular}

An $\mathrm{H}(4,6)$

\begin{tabular}{|l|l|l|l|}
\hline 15 & 26 & 37 & 48 \\
\hline 47 & 38 & 25 & 16 \\
\hline 28 & 17 & 46 & 35 \\
\hline 36 & 45 & 18 & 27 \\
\hline
\end{tabular}

An $\mathrm{H}^{*}(4,8)$ 
A $d$-dimensional Howell design $H_{d}(s, 2 n)$ is a $d$-dimensional array in which every cell either is empty or contains an unordered pair of symbols from an $s$-set and such that each two-dimensional projection is an $H(s, 2 n)$. An $H_{3}(s, 2 n)$ is a Howell cube. An $H_{d}(s, 2 n)$ is equivalent to $d$ mutually orthogonal 1-factorizations of the underlying graph (an $s$ regular graph on $2 n$ vertices). $\nu(s, 2 n)$ denotes the maximum value of $d$ such that an $H_{d}(s, 2 n)$ exists. Information on $\nu(s, 2 n)$ can be found in [1].

In 1986, A. Rosa and D. Stinson [13] studied orthogonal 1-factorizations of $s$-regular graphs on 10 or fewer vertices. In that paper it was claimed that there is no set of three orthogonal 1-factorizations of any 6-regular graph on 10 vertices. In other words, there is no Howell cube $H_{3}(6,10)$. This is not correct. Below we give three orthogonal 1 -factorizations, $F_{1}, F_{2}$ and $F_{3}$, of the graph which is the complement of $K_{4} \cup K_{3,3}$. Since this graph has an independent set of size four (namely $\{3,4,6,8\}$ ), the existence of these three orthogonal 1-factorizations implies there exists an $H_{3}^{*}(6,10)$.

Theorem 2.1 There exists an $H_{3}^{*}(6,10)$.

Proof: We display three orthogonal 1-factorizations of the 6-regular graph on 10 vertices which is the complement of $K_{4} \cup K_{3,3}$.

$$
F_{1} \quad F_{2}
$$

$\begin{array}{ll}\{1,10\},\{2,3\},\{4,9\},\{5,6\},\{7,8\} & \{1,3\},\{2,8\},\{4,7\},\{5,6\},\{9,10\} \\ \{1,3\},\{2,4\},\{5,7\},\{6,10\},\{8,9\} & \{1,10\},\{2,4\},\{3,9\},\{5,8\},\{6,7\} \\ \{1,4\},\{2,6\},\{3,7\},\{5,8\},\{9,10\} & \{1,9\},\{2,6\},\{3,10\},\{4,5\},\{7,8\} \\ \{1,6\},\{2,7\},\{3,9\},\{4,5\},\{8,10\} & \{1,8\},\{2,7\},\{3,5\},\{4,9\},\{6,10\} \\ \{1,9\},\{2,8\},\{3,5\},\{4,10\},\{6,7\} & \{1,4\},\{2,3\},\{5,7\},\{6,9\},\{8,10\} \\ \{1,8\},\{2,5\},\{3,10\},\{4,7\},\{6,9\} & \{1,6\},\{2,5\},\{3,7\},\{4,10\},\{8,9\}\end{array}$

\section{$F_{3}$}

$\{1,10\},\{8,9\},\{2,6\},\{3,5\},\{4,7\}$

$\{2,3\},\{9,10\},\{4,5\},\{6,7\},\{1,8\}$

$\{4,9\},\{5,7\},\{1,6\},\{2,8\},\{3,10\}$

$\{5,6\},\{2,4\},\{3,7\},\{8,10\},\{1,9\}$

$\{7,8\},\{6,10\},\{1,4\},\{3,9\},\{2,5\}$

$\{1,3\},\{5,8\},\{2,7\},\{4,10\},\{6,9\}$ 


\section{References}

[1] J. H. Dinitz. Howell Designs in The Handbook of Combinatorial Designs (2nd Edition), (C. J. Colbourn and J. H. Dinitz, eds.) Chapman \& Hall/CRC, Boca Raton, FL, 2007, 499-504.

[2] J. H. Dinitz and E. R. Lamken. Uniform Room frames with five holes. J. Combin. Designs 1 (1993), 323-328.

[3] J. H. Dinitz and D. R. Stinson. Further results on frames. Ars Combinatoria 11 (1981), 275-288.

[4] J. H. Dinitz And D. R. Stinson. A hill-climbing algorithm for the construction of one-factorizations and Room squares. SIAM J. on Algebraic and Discrete Methods 8 (1987), 430-438.

[5] J. H. Dinitz And D. R. Stinson. Room squares and related designs. In "Contemporary Design Theory: A Collection of Surveys", John Wiley \& Sons, 1992, pp. $137-204$.

[6] J. H. Dinitz, D. R. Stinson And L. Zhu. On the spectra of certain classes of Room frames, Electron. J. Combin. 1 (1994), Research Paper 7, 21 pp

[7] B. Du And L. Zhu. The existence of incomplete Room squares. J. Combin. Math. Combin. Comput. 14 (1993), 183-192.

[8] G. Ge. On the existence of Room frames of type $2^{n} u^{1}$. J. Statist. Plan. Infer., 94 (2001), 219-230.

[9] G. Ge And L. Zhu. On the existence of Room frames of type $t^{u}$ for $u=4$ and 5 . J. Combin. Designs 1 (1993), 183-191.

[10] G. Ge And L. Zhu. On the existence of Room frames of type $2^{n} u^{1}$. J. Combin. Math. Combin. Comput. 18 (1995), 65-82.

[11] P. B. Gibbons and Patric R. J. Östergånd, Computational methods in design theory in The Handbook of Combinatorial Designs (2nd Edition), (C. J. Colbourn and J. H. Dinitz, eds.) Chapman \& Hall/CRC, Boca Raton, FL, 2007, 755-782.

[12] R. C. Mullin And W. D. Wallis. The existence of Room squares. Aequationes Math. 13 (1975), 1-7.

[13] A. Rosa And D. R. Stinson. One-factorizations of regular graphs and Howell designs of small orders Utilitas Math. 29 (1986), 99-124.

[14] D. R. Stinson And L. Zhu. Towards the spectrum of Room squares with subsquares. J. Combin. Theory A 63 (1993), 129-142. 


\section{Appendix}

\section{A $(67,21)$-incomplete Room square}

$F_{r}$

$\begin{array}{lllllllllllllllllllllllllll}22,43 & 23,65 & 24,42 & 25,59 & 26,44 & 27,53 & 28,48 & 29,55 & 30,58 & 31,40 & 32,36 & 33,63 & 34,45 & 35,51 & 37,39 & 38,47 & 41,66 & 46,54 & 49,62 & 50,67 & 52,64 & 56,57 & 60,61\end{array}$

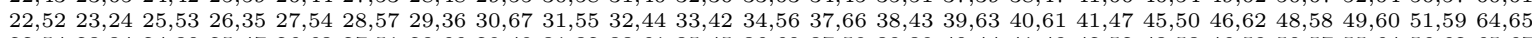

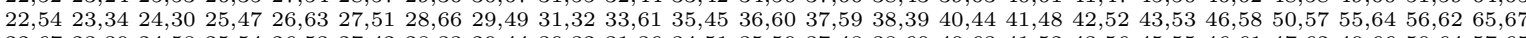

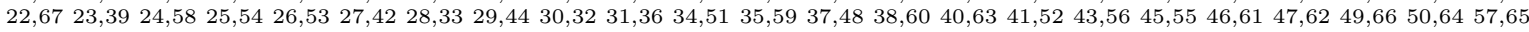
$\begin{array}{lllllllllllllllllllllllllll}22,40 & 23,44 & 24,33 & 25,48 & 26,54 & 27,47 & 28,49 & 29,31 & 30,59 & 32,58 & 34,52 & 35,65 & 36,63 & 37,67 & 38,42 & 39,53 & 41,55 & 43,60 & 45,51 & 46,57 & 50,62 & 56,61 & 64,66\end{array}$ $\begin{array}{lllllllllllllllllllllllll}22,36 & 23,45 & 24,65 & 25,58 & 26,60 & 27,48 & 28,61 & 29,30 & 31,50 & 32,55 & 33,37 & 34,42 & 35,39 & 38,44 & 40,54 & 41,49 & 43,51 & 46,59 & 47,52 & 53,62 & 56,66 & 57,63 & 64,67\end{array}$ $\begin{array}{llllllllllllllllllllllllllllll}22,33 & 23,57 & 24,31 & 25,49 & 26,46 & 27,45 & 28,35 & 29,39 & 30,64 & 32,61 & 34,41 & 36,38 & 37,54 & 40,62 & 42,59 & 43,44 & 47,67 & 48,51 & 50,55 & 52,66 & 53,65 & 56,58 & 60,63\end{array}$ $\begin{array}{llllllllllllllllllllllllllll}22,23 & 24,62 & 25,27 & 26,39 & 28,59 & 29,37 & 30,46 & 31,43 & 32,56 & 33,60 & 34,53 & 35,50 & 36,48 & 38,66 & 40,67 & 41,64 & 42,54 & 44,63 & 45,52 & 47,65 & 49,58 & 51,55 & 57,61\end{array}$ $\begin{array}{llllllllllllllllllllllllll}22,30 & 23,46 & 24,44 & 25,26 & 27,37 & 28,63 & 29,53 & 31,56 & 32,67 & 33,52 & 34,65 & 35,48 & 36,54 & 38,59 & 39,57 & 40,58 & 41,42 & 43,62 & 45,49 & 47,66 & 50,51 & 55,61 & 60,64\end{array}$ $\begin{array}{lllllllllllllllllllllllllllll}22,30 & 23,46 & 24,44 & 25,26 & 27,37 & 28,63 & 29,53 & 31,56 & 32,67 & 33,52 & 34,65 & 35,48 & 36,54 & 38,59 & 39,57 & 40,58 & 41,42 & 43,62 & 45,49 & 47,66 & 50,51 & 55,61 & 60,64 \\ 22,62 & 23,53 & 24,38 & 25,51 & 26,52 & 27,49 & 28,40 & 29,34 & 30,61 & 31,63 & 32,45 & 33,66 & 35,37 & 36,67 & 39,56 & 41,46 & 42,50 & 43,55 & 44,64 & 47,60 & 48,65 & 54,58 & 57,59\end{array}$ $\begin{array}{llllllllllllllllllllllllllllllll}22,62 & 23,53 & 24,38 & 25,51 & 26,52 & 27,49 & 28,40 & 29,34 & 30,61 & 31,63 & 32,45 & 33,66 & 35,37 & 36,67 & 39,56 & 41,46 & 42,50 & 43,55 & 44,64 & 47,60 & 48,65 & 54,58 & 57,59 \\ 22,37 & 23,60 & 24,64 & 25,38 & 26,56 & 27,30 & 28,41 & 29,51 & 31,66 & 32,47 & 33,35 & 34,40 & 36,55 & 39,46 & 42,45 & 43,65 & 44,57 & 48,67 & 49,53 & 50,63 & 52,61 & 54,62 & 58,59\end{array}$

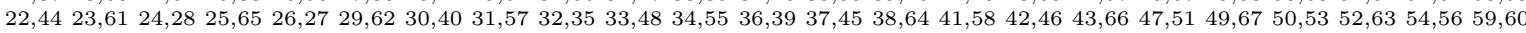
$\begin{array}{lllllllllllllllllllllllllllllllllll}22,47 & 23,31 & 24,56 & 25,42 & 26,37 & 27,66 & 28,51 & 29,46 & 30,48 & 32,49 & 33,41 & 34,43 & 35,57 & 36,59 & 38,54 & 39,58 & 40,64 & 44,65 & 45,63 & 50,52 & 53,61 & 55,62 & 60,67\end{array}$ $\begin{array}{llllllllllllllllllllllllllll}22,25 & 23,35 & 24,27 & 26,38 & 28,47 & 29,65 & 30,57 & 31,53 & 32,50 & 33,55 & 34,48 & 36,37 & 39,45 & 40,56 & 41,67 & 42,63 & 43,61 & 44,49 & 46,64 & 51,54 & 52,60 & 58,66 & 59,62\end{array}$ $\begin{array}{llllllllllllllllllllllllllll}22,66 & 23,25 & 24,37 & 26,61 & 27,57 & 28,53 & 29,47 & 30,33 & 31,52 & 32,41 & 34,50 & 35,63 & 36,42 & 38,40 & 39,64 & 43,48 & 44,46 & 45,56 & 49,55 & 51,67 & 54,59 & 58,60 & 62,65\end{array}$ $\begin{array}{llllllllllllllllllllllllllllllllllll}22,57 & 23,47 & 24,52 & 25,62 & 26,59 & 27,44 & 28,31 & 29,33 & 30,49 & 32,66 & 34,63 & 35,41 & 36,61 & 37,43 & 38,51 & 39,40 & 42,53 & 45,58 & 46,67 & 48,50 & 54,60 & 55,65 & 56,64\end{array}$

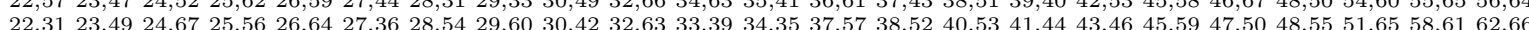

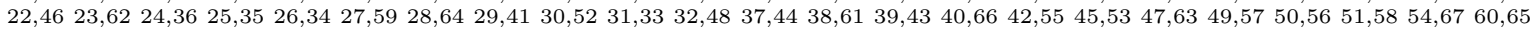

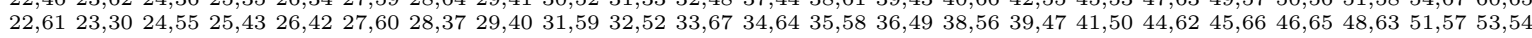

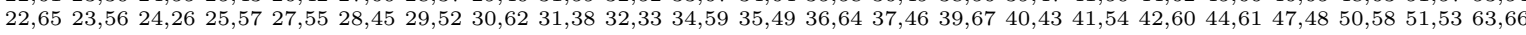
$\begin{array}{lllllllllllllllllllllllllllllll}22,64 & 23,29 & 24,40 & 25,45 & 26,32 & 27,43 & 28,56 & 30,51 & 31,39 & 33,46 & 34,49 & 35,38 & 36,44 & 37,63 & 41,65 & 42,66 & 47,61 & 48,59 & 50,60 & 52,53 & 54,55 & 57,67 & 58,62\end{array}$

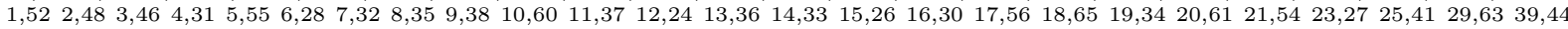
$40,5742,4345,6247,4950,5951,6453,6658,67$

$\begin{array}{llllllllllllllllllllllllllllllll}1,36 & 2,31 & 3,59 & 4,55 & 5,40 & 6,45 & 7,54 & 8,67 & 9,53 & 10,22 & 11,32 & 12,25 & 13,39 & 14,48 & 15,62 & 16,64 & 17,37 & 18,46 & 19,60 & 20,34 & 21,33 & 24,29 & 26,50 & 27,41 & 28,30\end{array}$ $35,5238,6342,5643,5744,5147,5849,6165,66$

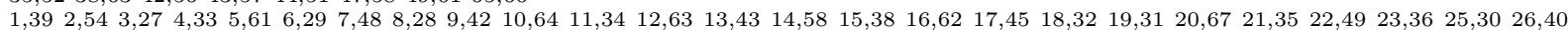
$37,5041,5144,4746,52 \quad 53,5556,6057,6659,65$

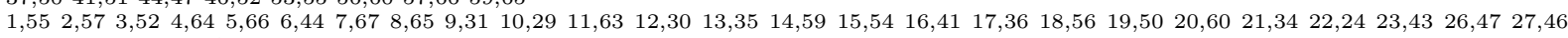
$28,5832,5133,38 \quad 37,6239,4940,45 \quad 42,6148,53$

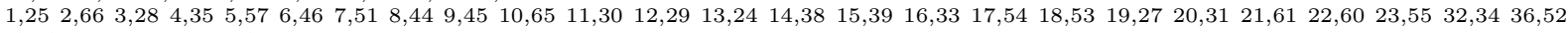
$37,4740,48 \quad 41,6242,6743,5049,5658,6359,64$

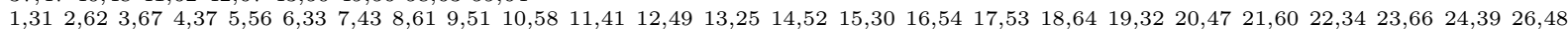
$28,38 \quad 29,35 \quad 36,57 \quad 40,5942,65 \quad 44,5045,46 \quad 55,63$

$\begin{array}{llllllllllllllllllllllllllllllllllllllll}1,54 & 2,51 & 3,65 & 4,26 & 5,63 & 6,32 & 7,53 & 8,46 & 9,61 & 10,27 & 11,43 & 12,57 & 13,49 & 14,55 & 15,25 & 16,58 & 17,34 & 18,39 & 19,37 & 20,64 & 21,67 & 22,41 & 23,42 & 24,35 & 29,56\end{array}$ $30,4431,4733,5036,4538,48,40,5259,66 \quad 60,62$

$\begin{array}{lllllllllllllllllllllllllllllllll}1,58 & 2,33 & 3,26 & 4,56 & 5,59 & 6,43 & 7,64 & 8,37 & 9,50 & 10,63 & 11,36 & 12,42 & 13,27 & 14,49 & 15,23 & 16,67 & 17,22 & 18,60 & 19,61 & 20,35 & 21,51 & 24,54 & 25,66 & 28,34 & 30,47\end{array}$ $31,4532,3938,5540,46 \quad 41,53 \quad 44,48 \quad 52,65 \quad 57,62$

$\begin{array}{llllllllllllllllllllllllllllllll}1,64 & 2,49 & 3,36 & 4,66 & 5,25 & 6,65 & 7,34 & 8,57 & 9,39 & 10,24 & 11,33 & 12,28 & 13,32 & 14,53 & 15,60 & 16,29 & 17,55 & 18,62 & 19,40 & 20,56 & 21,59 & 22,50 & 23,51 & 26,58 & 27,38\end{array}$ $31,3537,6141,4542,4743,67 \quad 44,5246,4854,63$

$\begin{array}{lllllllllllllllllllllllllllllllllll}1,35 & 2,26 & 3,41 & 4,54 & 5,46 & 6,34 & 7,56 & 8,40 & 9,55 & 10,47 & 11,45 & 12,37 & 13,59 & 14,60 & 15,24 & 16,61 & 17,52 & 18,49 & 19,43 & 20,23 & 21,25 & 22,63 & 27,58 & 28,29 & 30,39\end{array}$ $32,3833,5736,6542,48 \quad 44,6750,6651,6253,64$

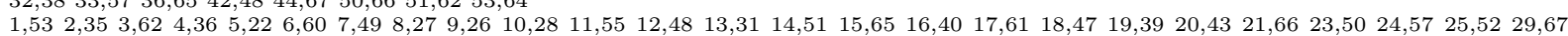
$30,63 \quad 33,34 \quad 37,6438,46 \quad 41,56 \quad 42,58 \quad 44,5945,54$

$\begin{array}{llllllllllllllllllllllllllllllllll}1,29 & 2,32 & 3,39 & 4,22 & 5,41 & 6,53 & 7,59 & 8,64 & 9,65 & 10,42 & 11,66 & 12,58 & 13,28 & 14,40 & 15,36 & 16,47 & 17,43 & 18,51 & 19,38 & 20,30 & 21,26 & 23,52 & 24,50 & 25,34 & 27,62\end{array}$ $31,5435,4437,4945,6746,6048,5655,5761,63$

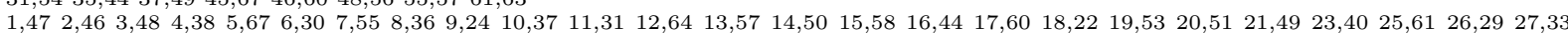
$28,3235,4239,4143,5945,65 \quad 52,5654,6662,63$

$\begin{array}{llllllllllllllllllllllllllll}1,61 & 2,40 & 3,53 & 4,27 & 5,42 & 6,26 & 7,22 & 8,39 & 9,36 & 10,25 & 11,51 & 12,43 & 13,46 & 14,31 & 15,44 & 16,60 & 17,63 & 18,24 & 19,28 & 20,50 & 21,58 & 23,67 & 29,64 & 30,56 & 32,62\end{array}$ $33,6534,6637,5238,4145,57 \quad 47,5548,5449,59$

$\begin{array}{llllllllllllllllllllllllllllllllllllll}1,65 & 2,43 & 3,49 & 4,50 & 5,26 & 6,37 & 7,46 & 8,60 & 9,58 & 10,67 & 11,59 & 12,41 & 13,38 & 14,44 & 15,42 & 16,56 & 17,62 & 18,29 & 19,22 & 20,52 & 21,45 & 23,63 & 24,34 & 25,32 & 27,40\end{array}$ $28,3930,6631,48 \quad 33,53 \quad 35,5547,57 \quad 51,6154,64$

$\begin{array}{llllllllllllllllllllllllllll}1,22 & 2,65 & 3,61 & 4,28 & 5,47 & 6,42 & 7,24 & 8,55 & 9,57 & 10,34 & 11,64 & 12,54 & 13,44 & 14,62 & 15,59 & 16,31 & 17,29 & 18,36 & 19,51 & 20,26 & 21,23 & 25,33 & 27,63 & 30,35 & 32,53\end{array}$ $38,5839,5240,5041,6043,4546,5648,4966,67$

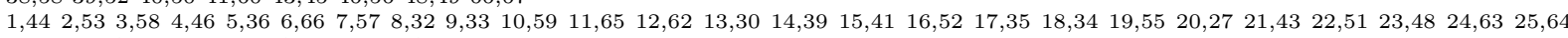
$26,4528,6729,5031,6037,4042,4947,56 \quad 54,61$

$\begin{array}{llllllllllllllllllllllllllll}1,56 & 2,34 & 3,38 & 4,67 & 5,58 & 6,24 & 7,66 & 8,54 & 9,29 & 10,51 & 11,48 & 12,33 & 13,23 & 14,65 & 15,52 & 16,26 & 17,47 & 18,59 & 19,36 & 20,28 & 21,44 & 22,45 & 25,63 & 27,61 & 30,50\end{array}$ $31,6432,4635,62 \quad 37,42 \quad 40,4941,43 \quad 53,57 \quad 55,60$

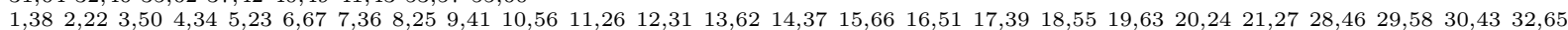
$33,5935,6042,6444,4547,5348,6149,5452,57$

$\begin{array}{llllllllllllllllllllllllllllllll}1,37 & 2,55 & 3,23 & 4,25 & 5,31 & 6,59 & 7,29 & 8,30 & 9,63 & 10,57 & 11,42 & 12,35 & 13,54 & 14,56 & 15,34 & 16,22 & 17,48 & 18,67 & 19,62 & 20,66 & 21,40 & 24,46 & 26,36 & 27,39 & 28,43\end{array}$ $32,6433,4738,6544,5345,6149,50 \quad 51,6052,58$

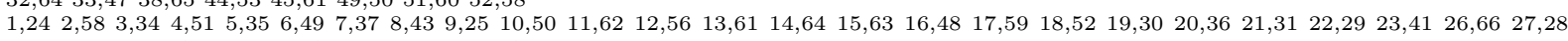
$32,6033,4538,6739,6540,47 \quad 44,5546,53 \quad 54,57$

$\begin{array}{llllllllllllllllllllllllllll}1,57 & 2,37 & 3,25 & 4,59 & 5,60 & 6,22 & 7,31 & 8,38 & 9,46 & 10,45 & 11,49 & 12,34 & 13,41 & 14,24 & 15,28 & 16,66 & 17,30 & 18,48 & 19,23 & 20,55 & 21,29 & 26,33 & 27,32 & 35,36 & 39,61\end{array}$ $40,5142,6244,5647,5450,6552,6753,5863,64$

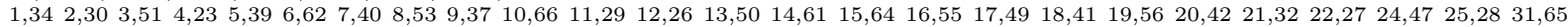
$33,3635,5438,4543,5846,6348,52 \quad 57,6059,67$

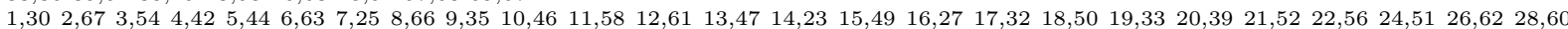
$29,43 \quad 31,4134,3736,53 \quad 38,5740,6548,6455,59$

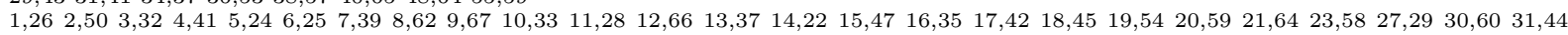
$34,6136,4338,5340,5548,5749,5251,5663,65$

$\begin{array}{llllllllllllllllllllllllllllllll}1,67 & 2,28 & 3,37 & 4,39 & 5,50 & 6,64 & 7,38 & 8,23 & 9,27 & 10,36 & 11,22 & 12,53 & 13,40 & 14,26 & 15,57 & 16,46 & 17,66 & 18,42 & 19,29 & 20,49 & 21,24 & 25,60 & 30,55 & 31,58 & 32,59\end{array}$ $33,4434,6235,56 \quad 41,6143,52 \quad 45,48 \quad 51,63 \quad 54,65$

$\begin{array}{lllllllllllllllllllllllllllll}1,66 & 2,63 & 3,35 & 4,61 & 5,27 & 6,56 & 7,60 & 8,24 & 9,22 & 10,41 & 11,50 & 12,40 & 13,52 & 14,67 & 15,53 & 16,39 & 17,23 & 18,31 & 19,58 & 20,45 & 21,36 & 25,55 & 26,28 & 29,59 & 30,38\end{array}$ $32,5433,43 \quad 34,57 \quad 37,6542,44 \quad 46,47 \quad 49,51 \quad 62,64$

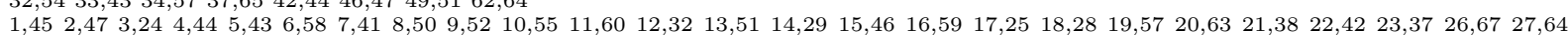
$30,6531,3433,4035,6136,62 \quad 39,54 \quad 48,6653,56$

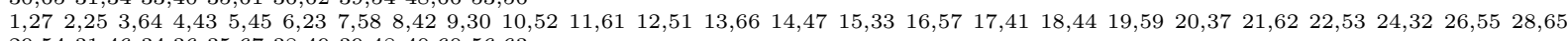
$29,5431,4634,3635,6738,4939,48 \quad 40,6056,63$

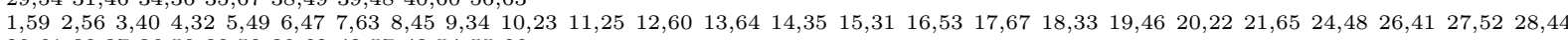
$29,6130,3736,5838,5039,62 \quad 42,57 \quad 43,5455,66$

$\begin{array}{llllllllllllllllllllllllll}1,46 & 2,44 & 3,63 & 4,45 & 5,30 & 6,27 & 7,33 & 8,49 & 9,32 & 10,61 & 11,54 & 12,65 & 13,58 & 14,43 & 15,29 & 16,50 & 17,28 & 18,57 & 19,35 & 20,62 & 21,48 & 22,38 & 23,26 & 24,66 & 25,40\end{array}$ 
$31,6734,6036,4137,5639,55 \quad 42,51 \quad 47,64 \quad 53,59$ $\begin{array}{llllllllllllllllllllllllllll}1,49 & 2,60 & 3,30 & 4,47 & 5,33 & 6,57 & 7,26 & 8,22 & 9,43 & 10,31 & 11,67 & 12,23 & 13,63 & 14,46 & 15,61 & 16,38 & 17,24 & 18,54 & 19,52 & 20,29 & 21,41 & 25,44 & 27,65 & 28,55 & 32,42\end{array}$ $34,3935,4036,5037,58 \quad 45,64 \quad 48,6251,66,56,59$

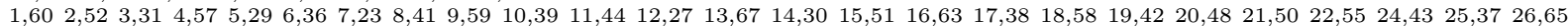
$28,6232,4033,5634,4635,5345,4749,6461,66$

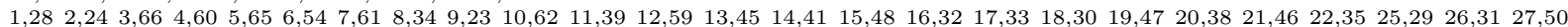
$36,5637,5340,4243,4944,5851,5257,6463,67$

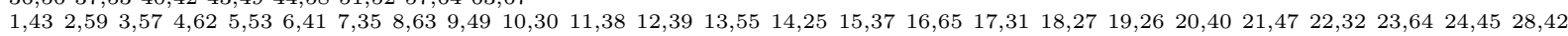
$29,4833,5834,4436,5146,50 \quad 52,5460,66 \quad 61,67$

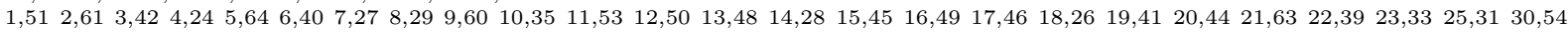
$32,43 \quad 34,4736,6637,3852,5955,5856,6562,67$

$\begin{array}{lllllllllllllllllllllllll}1,42 & 2,41 & 3,33 & 4,65 & 5,62 & 6,38 & 7,52 & 8,26 & 9,40 & 10,43 & 11,23 & 12,55 & 13,29 & 14,54 & 15,56 & 16,37 & 17,64 & 18,61 & 19,48 & 20,32 & 21,57 & 22,28 & 24,60 & 25,36 & 27,34\end{array}$ $30,4531,5135,4639,50 \quad 44,66 \quad 47,5949,63 \quad 53,67$

$\begin{array}{llllllllllllllllllllllllllllllllllllllll}1,41 & 2,38 & 3,22 & 4,58 & 5,48 & 6,31 & 7,62 & 8,51 & 9,66 & 10,26 & 11,57 & 12,52 & 13,33 & 14,27 & 15,32 & 16,23 & 17,40 & 18,35 & 19,64 & 20,25 & 21,55 & 24,49 & 28,50 & 29,45 & 30,34\end{array}$ $36,46 \quad 37,6039,42 \quad 43,4744,54 \quad 53,6356,6761,65$

$\begin{array}{lllllllllllllllllllllllllllllllll}1,40 & 2,29 & 3,43 & 4,52 & 5,28 & 6,51 & 7,44 & 8,47 & 9,64 & 10,48 & 11,27 & 12,45 & 13,53 & 14,42 & 15,22 & 16,36 & 17,65 & 18,38 & 19,24 & 20,58 & 21,56 & 23,32 & 25,50 & 26,57 & 30,31\end{array}$ $33,5434,6735,6637,5539,5941,6346,4961,62$

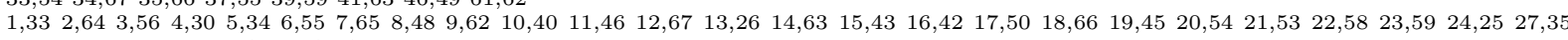
$28,5229,38 \quad 31,4932,57 \quad 36,47 \quad 37,4139,51 \quad 44,60$

$\begin{array}{lllllllllllllllllllllllllllll}1,50 & 2,42 & 3,29 & 4,63 & 5,52 & 6,61 & 7,45 & 8,31 & 9,48 & 10,38 & 11,24 & 12,44 & 13,22 & 14,36 & 15,40 & 16,25 & 17,51 & 18,23 & 19,65 & 20,53 & 21,28 & 26,30 & 27,56 & 32,37 & 33,49\end{array}$ $34,5435,4739,6041,5943,6446,6655,6757,58$

$\begin{array}{llllllllllllllllllllllllllllllllllll}1,23 & 2,39 & 3,55 & 4,40 & 5,32 & 6,48 & 7,28 & 8,52 & 9,56 & 10,44 & 11,47 & 12,38 & 13,60 & 14,34 & 15,35 & 16,45 & 17,27 & 18,43 & 19,67 & 20,33 & 21,42 & 22,26 & 24,53 & 25,46 & 29,66\end{array}$ $30,3631,62 \quad 37,5141,5749,65 \quad 50,5458,6459,61$

$\begin{array}{llllllllllllllllllllllllllllllllll}1,32 & 2,27 & 3,47 & 4,29 & 5,38 & 6,50 & 7,30 & 8,33 & 9,44 & 10,53 & 11,40 & 12,36 & 13,42 & 14,57 & 15,67 & 16,28 & 17,26 & 18,25 & 19,49 & 20,65 & 21,37 & 22,48 & 23,54 & 24,41 & 31,61\end{array}$ $34,5835,4339,6645,6046,5152,6255,5659,63$

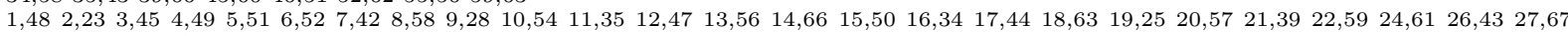
$29,3230,4131,3733,6436,4038,6246,55 \quad 53,60$

$\begin{array}{lllllllllllllllllllllllllllll}1,63 & 2,45 & 3,60 & 4,48 & 5,37 & 6,39 & 7,47 & 8,56 & 9,54 & 10,49 & 11,52 & 12,46 & 13,34 & 14,32 & 15,55 & 16,43 & 17,57 & 18,40 & 19,44 & 20,41 & 21,22 & 23,38 & 24,59 & 25,67 & 26,51\end{array}$ $27,3128,36 \quad 29,42 \quad 30,53 \quad 33,62 \quad 35,6450,6158,65$

$\begin{array}{llllllllllllllllllllllllllllll}1,62 & 2,36 & 3,44 & 4,53 & 5,54 & 6,35 & 7,50 & 8,59 & 9,47 & 10,32 & 11,56 & 12,22 & 13,65 & 14,45 & 15,27 & 16,24 & 17,58 & 18,37 & 19,66 & 20,46 & 21,30 & 23,28 & 25,39 & 26,49 & 29,57\end{array}$ $31,4233,5134,3840,4143,63 \quad 48,60 \quad 52,5561,64$

$F$

$\begin{array}{lllllllllllllllllllllll}22,45 & 23,64 & 24,46 & 25,39 & 26,31 & 27,38 & 28,60 & 29,54 & 30,43 & 32,37 & 33,62 & 34,58 & 35,53 & 36,40 & 41,57 & 42,61 & 44,59 & 47,49 & 48,52 & 50,65 & 51,63 & 55,66 & 56,67\end{array}$ $\begin{array}{llllllllllllllllllllllllllll}22,29 & 23,58 & 24,50 & 25,40 & 26,48 & 27,67 & 28,43 & 30,55 & 31,60 & 32,39 & 33,59 & 34,44 & 35,54 & 36,53 & 37,65 & 38,49 & 41,63 & 42,56 & 45,62 & 46,52 & 47,57 & 51,66 & 61,64\end{array}$ $\begin{array}{lllllllllllllllllllllllllll}22,32 & 23,51 & 24,61 & 25,64 & 26,55 & 27,31 & 28,44 & 29,38 & 30,39 & 33,50 & 34,54 & 35,40 & 36,57 & 37,52 & 41,43 & 42,58 & 45,67 & 46,53 & 47,59 & 48,66 & 49,65 & 56,60 & 62,63\end{array}$ $\begin{array}{llllllllllllllllllllllllll}22,34 & 23,33 & 24,49 & 25,32 & 26,66 & 27,61 & 28,29 & 30,63 & 31,67 & 35,42 & 36,51 & 37,40 & 38,65 & 39,54 & 41,45 & 43,52 & 44,60 & 46,56 & 47,55 & 48,53 & 50,59 & 57,62 & 58,64\end{array}$ $\begin{array}{llllllllllllllllllllllllllllllllll}22,58 & 23,63 & 24,29 & 25,30 & 26,51 & 27,52 & 28,42 & 31,62 & 32,54 & 33,34 & 35,67 & 36,41 & 37,60 & 38,55 & 39,61 & 40,65 & 43,49 & 44,50 & 45,47 & 46,66 & 48,57 & 53,64 & 56,59\end{array}$ $\begin{array}{lllllllllllllllllllllllllllllllllllll}22,58 & 23,63 & 24,29 & 25,30 & 26,51 & 27,52 & 28,42 & 31,62 & 32,54 & 33,34 & 35,67 & 36,41 & 37,60 & 38,55 & 39,61 & 40,65 & 43,49 & 44,50 & 45,47 & 46,66 & 48,57 & 53,64 & 56,59 \\ 22,53 & 23,48 & 24,47 & 25,60 & 26,41 & 27,63 & 28,62 & 29,58 & 30,31 & 32,57 & 33,38 & 34,61 & 35,64 & 36,50 & 37,49 & 39,55 & 40,59 & 42,43 & 44,67 & 45,54 & 46,51 & 52,56 & 65,66\end{array}$ $\begin{array}{lllllllllllllllllllllllllllllll}22,53 & 23,48 & 24,47 & 25,60 & 26,41 & 27,63 & 28,62 & 29,58 & 30,31 & 32,57 & 33,38 & 34,61 & 35,64 & 36,50 & 37,49 & 39,55 & 40,59 & 42,43 & 44,67 & 45,54 & 46,51 & 52,56 & 65,66 \\ 22,26 & 23,52 & 24,35 & 25,36 & 27,41 & 28,55 & 29,61 & 30,38 & 31,44 & 32,65 & 33,51 & 34,66 & 37,50 & 39,48 & 40,47 & 42,62 & 43,59 & 45,60 & 46,63 & 49,56 & 53,57 & 54,64 & 58,67\end{array}$ $\begin{array}{llllllllllllllllllllllllllllllll}22,38 & 23,67 & 24,54 & 25,44 & 26,65 & 27,62 & 28,50 & 29,43 & 30,45 & 31,37 & 32,53 & 33,36 & 34,47 & 35,56 & 39,41 & 40,60 & 42,48 & 46,49 & 51,61 & 52,58 & 55,63 & 57,66 & 59,64\end{array}$ $\begin{array}{lllllllllllllllllllllllllllll}22,41 & 23,50 & 24,25 & 26,28 & 27,65 & 29,42 & 30,60 & 31,34 & 32,64 & 33,45 & 35,46 & 36,66 & 37,51 & 38,57 & 39,44 & 40,49 & 43,67 & 47,54 & 48,56 & 52,55 & 53,59 & 58,63 & 61,62\end{array}$ $\begin{array}{lllllllllllllllllllllllllllllllll}22,35 & 23,66 & 24,45 & 25,41 & 26,62 & 27,40 & 28,30 & 29,67 & 31,64 & 32,34 & 33,65 & 36,43 & 37,61 & 38,50 & 39,60 & 42,51 & 44,48 & 46,55 & 47,56 & 49,63 & 52,59 & 53,58 & 54,57\end{array}$ $\begin{array}{llllllllllllllllllllllllllllll}22,48 & 23,40 & 24,57 & 25,63 & 26,43 & 27,64 & 28,52 & 29,59 & 30,66 & 31,46 & 32,51 & 33,47 & 34,37 & 35,36 & 38,45 & 39,42 & 41,53 & 44,58 & 49,61 & 50,54 & 55,67 & 56,65 & 60,62\end{array}$ $\begin{array}{lllllllllllllllllllllllllll}22,56 & 23,55 & 24,60 & 25,34 & 26,57 & 27,32 & 28,67 & 29,48 & 30,41 & 31,58 & 33,53 & 35,47 & 36,62 & 37,38 & 39,49 & 40,52 & 42,64 & 43,54 & 44,51 & 45,46 & 50,66 & 59,63 & 61,65\end{array}$

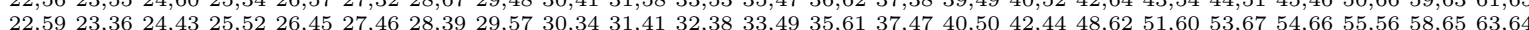
$\begin{array}{lllllllllllllllllllllllllllllll}22,24 & 23,59 & 25,67 & 26,40 & 27,34 & 28,38 & 29,50 & 30,65 & 31,42 & 32,46 & 33,44 & 35,66 & 36,45 & 37,53 & 39,62 & 41,60 & 43,57 & 47,64 & 48,61 & 49,51 & 52,54 & 55,58 & 56,63\end{array}$ $\begin{array}{llllllllllllllllllllllllllllllllll}22,24 & 23,59 & 25,67 & 26,40 & 27,34 & 28,38 & 29,50 & 30,65 & 31,42 & 32,46 & 33,44 & 35,66 & 36,45 & 37,53 & 39,62 & 41,60 & 43,57 & 47,64 & 48,61 & 49,51 & 52,54 & 55,58 & 56,63 \\ 22,50 & 23,43 & 24,32 & 25,28 & 26,33 & 27,39 & 29,66 & 30,35 & 31,47 & 34,57 & 36,58 & 37,42 & 38,46 & 40,55 & 41,59 & 44,45 & 48,60 & 49,64 & 51,52 & 53,56 & 54,65 & 61,63 & 62,67\end{array}$

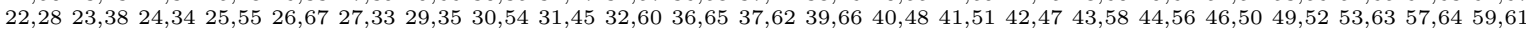
$\begin{array}{llllllllllllllllllllllllllllll}22,39 & 23,28 & 24,48 & 25,33 & 26,36 & 27,50 & 29,45 & 30,53 & 31,61 & 32,40 & 34,60 & 35,62 & 37,58 & 38,41 & 42,49 & 43,64 & 44,66 & 46,47 & 51,56 & 52,65 & 54,63 & 55,57 & 59,67\end{array}$ $\begin{array}{lllllllllllllllllllllllllllll}22,42 & 23,32 & 24,53 & 25,66 & 26,47 & 27,29 & 28,65 & 30,37 & 31,54 & 33,56 & 34,39 & 35,60 & 36,52 & 38,62 & 40,41 & 43,45 & 44,55 & 46,48 & 49,59 & 50,61 & 51,64 & 57,58 & 63,67\end{array}$ $\begin{array}{llllllllllllllllllllllllllllllll}22,51 & 23,27 & 24,59 & 25,31 & 26,49 & 28,32 & 29,56 & 30,36 & 33,43 & 34,62 & 35,55 & 37,41 & 38,58 & 39,50 & 40,45 & 42,57 & 44,54 & 46,60 & 47,53 & 48,64 & 52,67 & 61,66 & 63,65\end{array}$ $\begin{array}{lllllllllllllllllllllllllllllllll}22,63 & 23,42 & 24,51 & 25,29 & 26,50 & 27,56 & 28,46 & 30,47 & 31,49 & 32,59 & 33,58 & 34,36 & 35,43 & 37,64 & 38,53 & 39,65 & 40,57 & 41,62 & 44,52 & 45,61 & 48,54 & 55,60 & 66,67\end{array}$ $\begin{array}{llllllllllllllllllllllllllllll}22,60 & 23,26 & 24,41 & 25,37 & 27,58 & 28,34 & 29,32 & 30,56 & 31,35 & 33,40 & 36,46 & 38,67 & 39,51 & 42,65 & 43,63 & 44,47 & 45,48 & 49,50 & 52,57 & 53,66 & 54,61 & 55,59 & 62,64\end{array}$

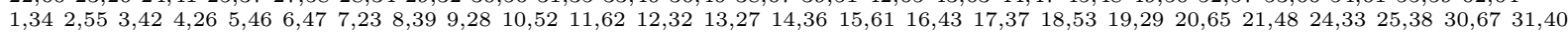
$35,6341,4445,6649,5850,5651,5457,5960,64$

$\begin{array}{lllllllllllllllllllllllllll}1,28 & 2,51 & 3,30 & 4,61 & 5,36 & 6,49 & 7,29 & 8,45 & 9,47 & 10,25 & 11,58 & 12,66 & 13,48 & 14,44 & 15,50 & 16,33 & 17,57 & 18,42 & 19,53 & 20,26 & 21,35 & 22,27 & 24,31 & 32,41 & 34,65\end{array}$ $37,5638,6039,6340,6443,5546,5952,6254,67$

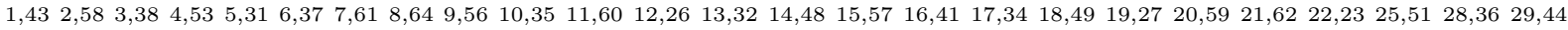
$30,4033,4239,4546,6547,6350,6752,6654,55$

$\begin{array}{llllllllllllllllllllllllllllllll}1,30 & 2,49 & 3,60 & 4,43 & 5,42 & 6,59 & 7,57 & 8,52 & 9,37 & 10,47 & 11,26 & 12,34 & 13,44 & 14,33 & 15,62 & 16,23 & 17,53 & 18,32 & 19,58 & 20,46 & 21,67 & 22,31 & 24,28 & 27,35 & 29,40\end{array}$ $36,5438,6639,5641,6145,6548,51 \quad 50,6355,64$

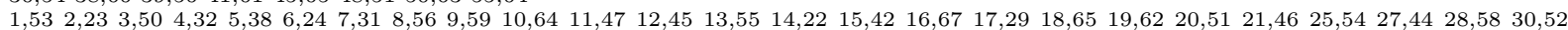
$33,3934,4035,3736,48 \quad 41,4943,6157,6063,66$

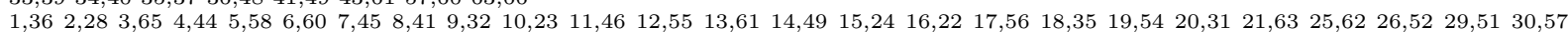
$33,6734,53 \quad 37,6638,5939,43 \quad 40,42 \quad 47,48 \quad 50,64$

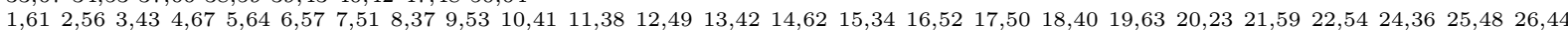
$27,45 \quad 29,3031,6532,66 \quad 33,55 \quad 35,58 \quad 39,46 \quad 47,60$

$\begin{array}{llllllllllllllllllllllllllllllll}1,38 & 2,54 & 3,45 & 4,62 & 5,27 & 6,32 & 7,49 & 8,43 & 9,55 & 10,53 & 11,63 & 12,64 & 13,40 & 14,31 & 15,33 & 16,36 & 17,42 & 18,61 & 19,51 & 20,48 & 21,28 & 22,25 & 23,39 & 24,44 & 26,59\end{array}$ $30,58 \quad 34,5235,5037,5741,4647,6756,66 \quad 60,65$

$\begin{array}{llllllllllllllllllllllllllllllllll}1,54 & 2,47 & 3,37 & 4,25 & 5,24 & 6,44 & 7,62 & 8,29 & 9,38 & 10,51 & 11,50 & 12,61 & 13,31 & 14,40 & 15,22 & 16,56 & 17,52 & 18,48 & 19,67 & 20,53 & 21,27 & 23,49 & 26,35 & 28,63 & 32,42\end{array}$ $33,6634,5536,5939,6441,6543,6045,5746,58$

$\begin{array}{llllllllllllllllllllllllllllllllll}1,22 & 2,38 & 3,57 & 4,36 & 5,37 & 6,66 & 7,58 & 8,33 & 9,23 & 10,61 & 11,48 & 12,28 & 13,24 & 14,29 & 15,47 & 16,55 & 17,27 & 18,26 & 19,25 & 20,45 & 21,40 & 30,46 & 32,63 & 34,50 & 35,44\end{array}$ $39,5941,4243,5149,6752,53 \quad 54,6056,6264,65$

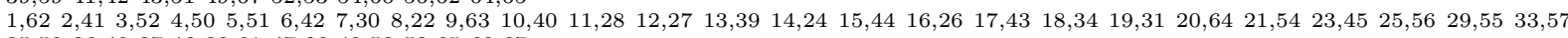
$35,5936,4937,4638,6147,66 \quad 48,5853,6560,67$

$\begin{array}{lllllllllllllllllllllllllllllll}1,59 & 2,64 & 3,25 & 4,42 & 5,67 & 6,48 & 7,54 & 8,60 & 9,50 & 10,43 & 11,45 & 12,46 & 13,66 & 14,57 & 15,32 & 16,30 & 17,58 & 18,38 & 19,65 & 20,63 & 21,36 & 22,62 & 23,61 & 24,26 & 27,47\end{array}$ $28,51 \quad 29,49 \quad 31,52 \quad 34,35 \quad 37,44 \quad 39,40 \quad 41,56 \quad 53,55$

$\begin{array}{llllllllllllllllllllllllllllllllllllll}1,56 & 2,62 & 3,32 & 4,45 & 5,40 & 6,23 & 7,59 & 8,66 & 9,25 & 10,49 & 11,36 & 12,53 & 13,63 & 14,60 & 15,51 & 16,58 & 17,55 & 18,29 & 19,50 & 20,54 & 21,37 & 22,52 & 24,67 & 26,42 & 27,43\end{array}$ $28,3130,6133,35 \quad 38,6439,57 \quad 41,48 \quad 44,46 \quad 47,65$

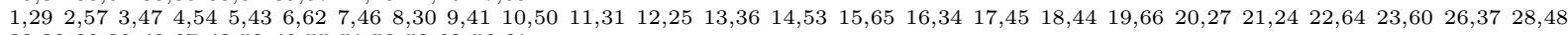
$32,3338,3940,6742,5949,55 \quad 51,58 \quad 52,6356,61$

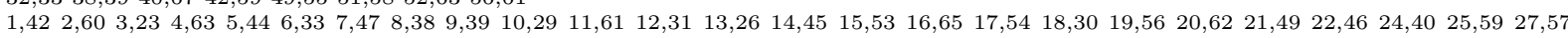
$28,3732,58 \quad 34,51 \quad 35,4143,66 \quad 48,55 \quad 50,52 \quad 64,67$

$\begin{array}{lllllllllllllllllllllllllll}1,47 & 2,52 & 3,56 & 4,34 & 5,41 & 6,29 & 7,28 & 8,49 & 9,44 & 10,31 & 11,24 & 12,48 & 13,46 & 14,35 & 15,63 & 16,54 & 17,25 & 18,45 & 19,55 & 20,39 & 21,33 & 22,40 & 23,57 & 26,38 & 27,42\end{array}$ $30,5032,43 \quad 36,6451,5953,6158,6062,66 \quad 65,67$

$\begin{array}{llllllllllllllllllllllllllll}1,49 & 2,34,22 & 4,30 & 5,47 & 6,65 & 7,39 & 8,46 & 9,45 & 10,48 & 11,25 & 12,43 & 13,52 & 14,56 & 15,58 & 16,24 & 17,66 & 18,28 & 19,59 & 20,67 & 21,31 & 23,44 & 26,61 & 27,60 & 29,37\end{array}$ $32,3533,6436,55 \quad 40,5141,54 \quad 42,63 \quad 50,57 \quad 53,62$ 
$\begin{array}{lllllllllllllllllllllllllllll}1,52 & 2,50 & 3,53 & 4,37 & 5,57 & 6,30 & 7,60 & 8,31 & 9,58 & 10,32 & 11,64 & 12,23 & 13,41 & 14,26 & 15,45 & 16,27 & 17,35 & 18,25 & 19,44 & 20,36 & 21,42 & 22,43 & 24,56 & 28,54 & 29,47\end{array}$ $33,4634,5938,6340,62 \quad 48,6549,66 \quad 51,5561,67$

$\begin{array}{lllllllllllllllllllllllllllll}1,39 & 2,33 & 3,49 & 4,29 & 5,25 & 6,56 & 7,67 & 8,50 & 9,60 & 10,44 & 11,55 & 12,52 & 13,64 & 14,42 & 15,26 & 16,66 & 17,32 & 18,58 & 19,28 & 20,41 & 21,47 & 22,61 & 23,35 & 24,30 & 27,53\end{array}$ $31,43 \quad 34,63 \quad 36,38 \quad 37,45 \quad 46,57 \quad 48,59 \quad 51,65 \quad 54,62$

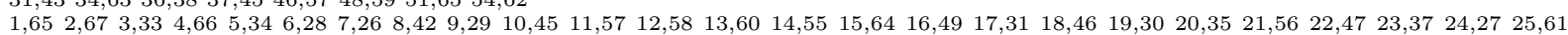

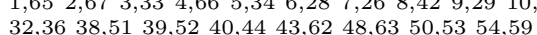

$\begin{array}{llllllllllllllllllllllllllllllllll}1,46 & 2,30 & 3,66 & 4,35 & 5,56 & 6,26 & 7,63 & 8,28 & 9,40 & 10,55 & 11,44 & 12,38 & 13,65 & 14,37 & 15,41 & 16,29 & 17,23 & 18,36 & 19,34 & 20,25 & 21,51 & 22,57 & 24,64 & 27,59 & 31,48\end{array}$ $32,5033,5439,6743,5345,4947,52 \quad 58,6260,61$

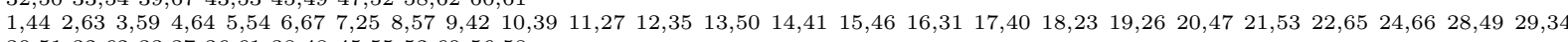
$30,5132,6233,3736,6138,48 \quad 45,5552,6056,58$

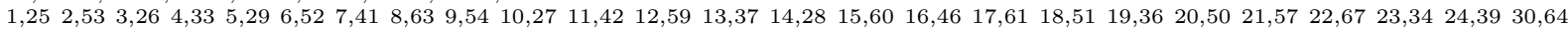
$31,6632,5535,3840,5643,4745,58 \quad 48,4962,65$

$\begin{array}{llllllllllllllllllllllllllll}1,64 & 2,27 & 3,61 & 4,47 & 5,32 & 6,54 & 7,56 & 8,51 & 9,26 & 10,42 & 11,30 & 12,65 & 13,29 & 14,63 & 15,31 & 16,48 & 17,62 & 18,59 & 19,23 & 20,60 & 21,39 & 22,37 & 24,55 & 25,50 & 28,33\end{array}$ $34,4635,5236,6738,43 \quad 40,5841,6644,5349,57$

$\begin{array}{llllllllllllllllllllllllllllllllllll}1,40 & 2,36 & 3,55 & 4,23 & 5,45 & 6,63 & 7,24 & 8,34 & 9,31 & 10,54 & 11,49 & 12,56 & 13,30 & 14,27 & 15,43 & 16,38 & 17,28 & 18,39 & 19,41 & 20,22 & 21,50 & 25,57 & 26,60 & 29,65 & 32,61\end{array}$ $33,5235,5137,5942,5344,6247,5848,6764,66$

$\begin{array}{llllllllllllllllllllllllllllll}1,35 & 2,59 & 3,27 & 4,56 & 5,26 & 6,58 & 7,32 & 8,48 & 9,24 & 10,22 & 11,51 & 12,57 & 13,62 & 14,66 & 15,52 & 16,50 & 17,41 & 18,33 & 19,38 & 20,29 & 21,30 & 23,65 & 25,43 & 28,45 & 31,39\end{array}$ $34,6736,6337,5440,5342,5544,6446,6149,60$

$\begin{array}{lllllllllllllllllllllllllllll}1,23 & 2,44 & 3,46 & 4,27 & 5,49 & 6,25 & 7,65 & 8,54 & 9,33 & 10,57 & 11,40 & 12,63 & 13,34 & 14,38 & 15,56 & 16,32 & 17,22 & 18,52 & 19,64 & 20,24 & 21,66 & 26,30 & 28,61 & 29,60 & 31,59\end{array}$ $35,45 \quad 36,42 \quad 37,43 \quad 39,53 \quad 41,67 \quad 47,51 \quad 50,58 \quad 55,62$

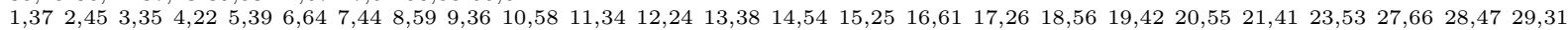
$30,62 \quad 32,52 \quad 33,48 \quad 40,4643,65 \quad 50,6051,6757,63$

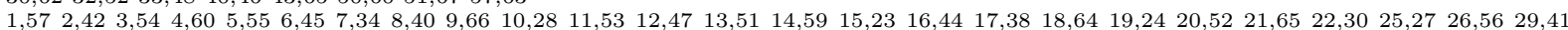
$31,3233,63 \quad 35,4936,3937,6743,48 \quad 46,6258,61$

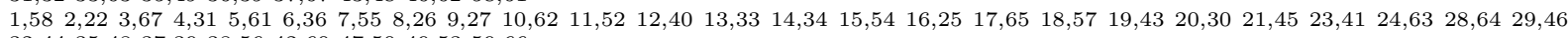
$32,4435,4837,3938,5642,60 \quad 47,5049,53 \quad 59,66$

$\begin{array}{lllllllllllllllllllllllllll}1,67 & 2,24 & 3,58 & 4,41 & 5,53 & 6,40 & 7,36 & 8,55 & 9,30 & 10,26 & 11,37 & 12,33 & 13,35 & 14,61 & 15,49 & 16,42 & 17,51 & 18,54 & 19,39 & 20,34 & 21,38 & 22,44 & 23,31 & 25,45 & 27,48\end{array}$ $28,6629,6432,4743,4650,6256,5759,6560,63$

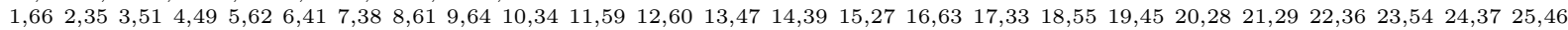
$26,5830,3231,5640,43 \quad 42,52 \quad 44,6548,50 \quad 57,67$

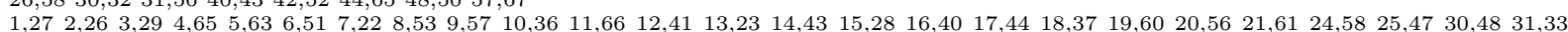
$32,45 \quad 34,4935,39 \quad 38,42 \quad 46,67 \quad 50,55 \quad 52,64 \quad 59,62$

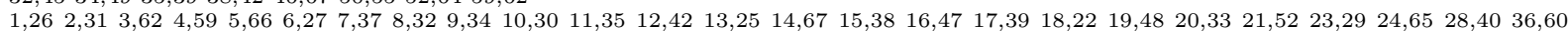
$41,5843,5044,4945,6346,5451,5356,6457,61$

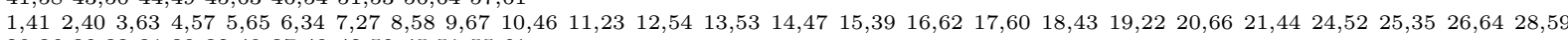
$29,3630,33 \quad 31,3832,4937,48 \quad 42,50 \quad 45,51 \quad 55,61$

$\begin{array}{lllllllllllllllllllllllllllll}1,45 & 2,43 & 3,39 & 4,40 & 5,28 & 6,50 & 7,35 & 8,44 & 9,48 & 10,37 & 11,65 & 12,67 & 13,59 & 14,32 & 15,30 & 16,53 & 17,63 & 18,41 & 19,52 & 20,49 & 21,64 & 22,55 & 23,25 & 24,42 & 26,46\end{array}$ $27,5129,6231,3633,6034,5638,54 \quad 47,6158,66$

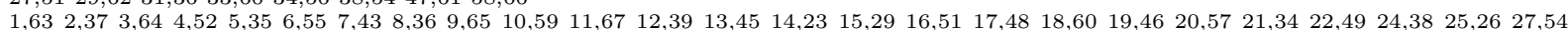
$28,53 \quad 30,42 \quad 31,5032,5633,4140,6644,6147,62$

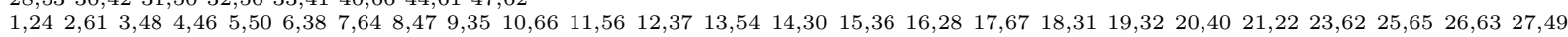
$29,33 \quad 34,4239,5841,5543,44 \quad 45,5251,5753,60$

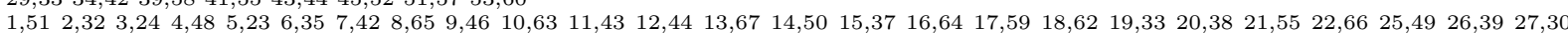
$28,5629,53 \quad 31,5734,45 \quad 36,4740,6141,52 \quad 54,58$

$\begin{array}{lllllllllllllllllllllllllll}1,31 & 2,39 & 3,28 & 4,58 & 5,22 & 6,53 & 7,40 & 8,67 & 9,52 & 10,38 & 11,33 & 12,30 & 13,56 & 14,65 & 15,48 & 16,57 & 17,24 & 18,50 & 19,35 & 20,32 & 21,43 & 23,46 & 25,42 & 26,27 & 29,63\end{array}$ $34,6436,4437,5541,4745,5949,5451,6260,66$

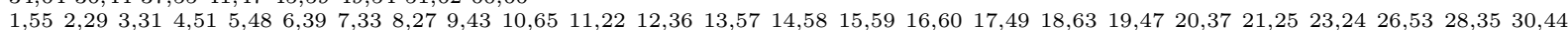
$32,6734,38 \quad 40,54 \quad 41,5042,6645,5646,6452,61$

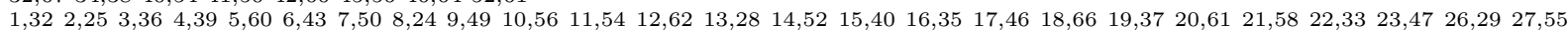
$30,5931,5134,48 \quad 38,4441,6442,6745,53 \quad 57,65$

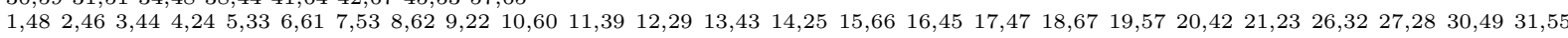
$34,4135,6536,3738,52 \quad 40,63 \quad 50,5154,5658,59$

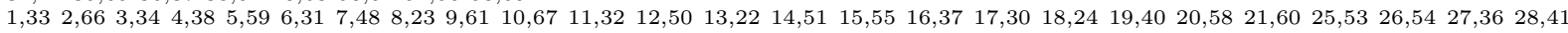
$29,5235,5739,4742,46 \quad 43,56 \quad 44,6345,6449,62$

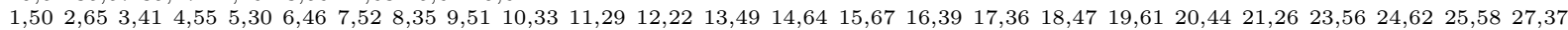
$28,5731,63 \quad 32,48 \quad 34,43 \quad 38,4042,45 \quad 53,5459,60$

$\begin{array}{llllllllllllllllllllllllllllllllll}1,60 & 2,48 & 3,40 & 4,28 & 5,52 & 6,22 & 7,66 & 8,25 & 9,62 & 10,24 & 11,41 & 12,51 & 13,58 & 14,46 & 15,35 & 16,59 & 17,64 & 18,27 & 19,49 & 20,43 & 21,32 & 23,30 & 26,34 & 29,39 & 31,53\end{array}$ $33,6136,5637,63 \quad 38,4742,5444,57 \quad 45,50 \quad 55,65$

\section{A Room frame of type $14^{4}$}

\section{$F_{r}$}

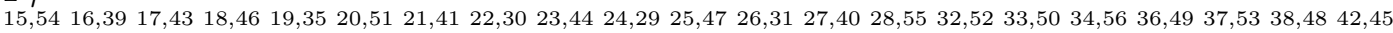
$\begin{array}{lllllllllllllllllllllllll}15,48 & 16,40 & 17,44 & 18,34 & 19,43 & 20,39 & 21,30 & 22,50 & 23,32 & 24,36 & 25,33 & 26,45 & 27,56 & 28,52 & 29,46 & 31,51 & 35,53 & 37,49 & 38,47 & 41,54 & 42,55\end{array}$ $\begin{array}{llllllllllllllllllllllllllll}15,50 & 16,47 & 17,46 & 18,51 & 19,38 & 20,29 & 21,45 & 22,43 & 23,41 & 24,42 & 25,36 & 26,39 & 27,53 & 28,33 & 30,56 & 31,55 & 32,49 & 34,44 & 35,52 & 37,54 & 40,48\end{array}$ $\begin{array}{lllllllllllllllllllllllll}15,55 & 16,46 & 17,56 & 18,33 & 19,41 & 20,36 & 21,32 & 22,38 & 23,54 & 24,34 & 25,45 & 26,35 & 27,43 & 28,50 & 29,51 & 30,44 & 31,47 & 37,52 & 39,48 & 40,53 & 42,49\end{array}$ $\begin{array}{llllllllllllllllllllllllll}15,43 & 16,29 & 17,45 & 18,32 & 19,54 & 20,49 & 21,52 & 22,39 & 23,33 & 24,51 & 25,50 & 26,36 & 27,34 & 28,42 & 30,46 & 31,44 & 35,48 & 37,55 & 38,53 & 40,56 & 41,47\end{array}$ $\begin{array}{lllllllllllllllllllllll}15,36 & 16,34 & 17,30 & 18,54 & 19,37 & 20,43 & 21,46 & 22,53 & 23,52 & 24,50 & 25,40 & 26,49 & 27,29 & 28,39 & 31,45 & 32,48 & 33,51,51,55 & 35,44 & 41,56 & 42,47\end{array}$ $\begin{array}{lllllllllllllllllllllllllllllllll}15,35 & 16,56 & 17,52 & 18,36 & 19,39 & 20,45 & 21,34 & 22,33 & 23,48 & 24,37 & 25,51 & 26,43 & 27,44 & 28,38 & 29,49 & 30,53 & 31,46 & 32,47 & 40,55 & 41,50 & 42,54\end{array}$

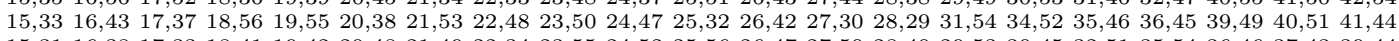
$\begin{array}{llllllllllllllllllllllllllllllllll}15,31 & 16,38 & 17,33 & 18,41 & 19,42 & 20,48 & 21,49 & 22,34 & 23,55 & 24,52 & 25,56 & 26,47 & 27,50 & 28,40 & 29,53 & 30,45 & 32,51 & 35,54 & 36,46 & 37,43 & 39,44\end{array}$

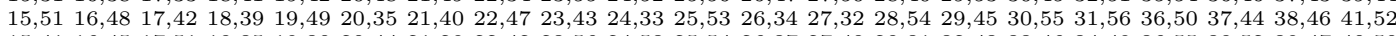
$\begin{array}{llllllllllllllllllllllllllll}15,41 & 16,45 & 17,51 & 18,35 & 19,30 & 20,44 & 21,29 & 22,42 & 23,56 & 24,53 & 25,54 & 26,37 & 27,48 & 28,31 & 32,43 & 33,46 & 34,49 & 36,55 & 38,52 & 39,47 & 40,50\end{array}$ $\begin{array}{llllllllllllllllllllllllll}15,49 & 16,32 & 17,53 & 18,55 & 19,45 & 20,50 & 21,54 & 22,44 & 23,40 & 24,41 & 25,29 & 26,38 & 27,35 & 28,30 & 31,43 & 33,52 & 34,51 & 36,56 & 37,47 & 39,46 & 42,48\end{array}$ $\begin{array}{lllllllllllllllllllllllllll}15,53 & 16,55 & 17,29 & 18,30 & 19,52 & 20,42 & 21,48 & 22,45 & 23,35 & 24,31 & 25,44 & 26,46 & 27,36 & 28,41 & 32,54 & 33,49 & 34,47 & 37,56 & 38,50 & 39,51 & 40,43\end{array}$ $\begin{array}{lllllllllllllllllllllllllllllll}15,38 & 16,50 & 17,49 & 18,52 & 19,46 & 20,55 & 21,36 & 22,32 & 23,30 & 24,45 & 25,39 & 26,54 & 27,31 & 28,37 & 29,47 & 33,48 & 34,43 & 35,51 & 40,44 & 41,53 & 42,56\end{array}$

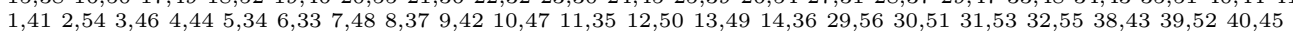
$\begin{array}{llllllllllllllllllllllll}1,39 & 2,30 & 3,34 & 4,55 & 5,49 & 6,32 & 7,53 & 8,46 & 9,37 & 10,48 & 11,31 & 12,38 & 13,45 & 14,50 & 29,52 & 33,44 & 35,56 & 36,54 & 40,47 & 41,43 & 42,51\end{array}$ $\begin{array}{lllllllllllllllllllllllllll}1,49 & 2,53 & 3,55 & 4,35 & 5,40 & 6,36 & 7,52 & 8,54 & 9,41 & 10,34 & 11,48 & 12,31 & 13,43 & 14,39 & 29,50 & 30,47 & 32,46 & 33,56 & 37,51 & 38,45 & 42,44\end{array}$ $\begin{array}{llllllllllllllllllllllll}1,48 & 2,37 & 3,47 & 4,29 & 5,46 & 6,51 & 7,34 & 8,44 & 9,53 & 10,31 & 11,30 & 12,39 & 13,56 & 14,41 & 32,50 & 33,55 & 35,45 & 36,43 & 38,54 & 40,49 & 42,52\end{array}$ $\begin{array}{lllllllllllllllllllllllll}1,37 & 2,47 & 3,50 & 4,31 & 5,51 & 6,52 & 7,45 & 8,35 & 9,49 & 10,33 & 11,40 & 12,42 & 13,54 & 14,38 & 29,44 & 30,43 & 32,56 & 34,46 & 36,48 & 39,53 & 41,55\end{array}$ $\begin{array}{lllllllllllllllllllllll}1,56 & 2,55 & 3,30 & 4,46 & 5,47 & 6,41 & 7,37 & 8,29 & 9,34 & 10,43 & 11,51 & 12,36 & 13,33 & 14,52 & 31,48 & 32,44 & 35,50 & 38,49 & 39,45 & 40,54 & 42,53\end{array}$ $\begin{array}{llllllllllllllllllllllllll}1,55 & 2,41 & 3,48 & 4,42 & 5,32 & 6,53 & 7,44 & 8,49 & 9,54 & 10,40 & 11,38 & 12,29 & 13,39 & 14,56 & 30,52 & 31,50 & 33,43 & 34,45 & 35,47 & 36,51 & 37,46\end{array}$ $\begin{array}{llllllllllllllllllllllllll}1,38 & 2,31 & 3,41 & 4,51 & 5,33 & 6,56 & 7,36 & 8,45 & 9,30 & 10,54 & 11,47 & 12,46 & 13,42 & 14,44 & 29,43 & 32,53 & 34,50 & 35,49 & 37,48 & 39,55 & 40,52\end{array}$

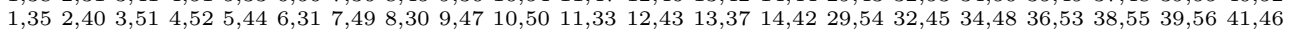
$\begin{array}{llllllllllllllllllllll}1,32 & 2,33 & 3,44 & 4,56 & 5,38 & 6,35 & 7,46 & 8,42 & 9,50 & 10,52 & 11,49 & 12,53 & 13,31 & 14,40 & 29,55 & 30,48 & 34,54 & 36,47 & 37,45 & 39,43 & 41,51\end{array}$ $\begin{array}{lllllllllllllllllllllllll}1,51 & 2,36 & 3,53 & 4,47 & 5,35 & 6,49 & 7,38 & 8,32 & 9,29 & 10,44 & 11,37 & 12,56 & 13,34 & 14,55 & 30,50 & 31,52 & 33,45 & 39,54 & 40,46 & 41,48 & 42,43\end{array}$ 
$\begin{array}{lllllllllllllllllllll}1,46 & 2,44 & 3,32 & 4,41 & 5,55 & 6,38 & 7,51 & 8,31 & 9,39 & 10,37 & 11,56 & 12,54 & 13,40 & 14,45 & 29,48 & 30,49 & 33,47 & 34,53 & 35,43 & 36,52 & 42,50\end{array}$ $\begin{array}{llllllllllllllllllllllll}1,42 & 2,32 & 3,29 & 4,39 & 5,56 & 6,43 & 7,40 & 8,34 & 9,48 & 10,55 & 11,46 & 12,52 & 13,35 & 14,47 & 30,54 & 31,49 & 33,53 & 36,44 & 37,50 & 38,51 & 41,45\end{array}$ $\begin{array}{lllllllllllllllllllllllll}1,29 & 2,48 & 3,52 & 4,53 & 5,36 & 6,37 & 7,30 & 8,43 & 9,40 & 10,32 & 11,45 & 12,47 & 13,51 & 14,31 & 33,54 & 34,55 & 35,44 & 38,56 & 39,50 & 41,49 & 42,46\end{array}$ $\begin{array}{llllllllllllllllllllllllll}1,21 & 2,26 & 3,54 & 4,23 & 5,16 & 6,18 & 7,56 & 8,47 & 9,44 & 10,28 & 11,22 & 12,51 & 13,52 & 14,53 & 15,45 & 17,50 & 19,48 & 20,46 & 24,49 & 25,43 & 27,55\end{array}$ $\begin{array}{lllllllllllllllllllllll}1,52 & 2,50 & 3,27 & 4,21 & 5,43 & 6,20 & 7,54 & 8,17 & 9,51 & 10,53 & 11,26 & 12,16 & 13,55 & 14,24 & 15,46 & 18,45 & 19,44 & 22,56 & 23,47 & 25,49 & 28,48\end{array}$ $\begin{array}{lllllllllllllllllllllllll}1,26 & 2,43 & 3,15 & 4,16 & 5 & 20 & 6,54 & 7,18 & 8,48 & 9,52 & 10,49 & 11,53 & 12,22 & 13,25 & 14,51 & 17,47 & 19,50 & 21,55 & 23,45 & 24,44 & 27,46 & 28,56\end{array}$ $\begin{array}{lllllllllllllllllllllll}1,26 & 2,43 & 3,15 & 4,16 & 5,20 & 6,54 & 7,18 & 8,48 & 9,52 & 10,49 & 11,53 & 12,22 & 13,25 & 14,51 & 17,47 & 19,50 & 21,55 & 23,45 & 24,44 & 27,46 & 28,56 \\ 1,54 & 2,52 & 3,56 & 4,17 & 5,45 & 6,25 & 7,21 & 8,22 & 9,46 & 10,19 & 11,50 & 12,27 & 13,15 & 14,43 & 16,53 & 18,44 & 20,47 & 23,51 & 24,55 & 26,48 & 28,49\end{array}$ $\begin{array}{lllllllllllllllllllllllllllllllll}1,50 & 2,49 & 3,16 & 4,27 & 5,48 & 6,45 & 7,20 & 8,23 & 9,19 & 10,17 & 11,54 & 12,55 & 13,53 & 14,25 & 15,52 & 18,43 & 21,56 & 22,51 & 24,46 & 26,44 & 28,47\end{array}$

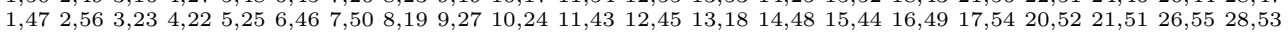
$\begin{array}{llllllllllllllllllllllllll}1,44 & 2,46 & 3,22 & 4,48 & 5,54 & 6,17 & 7,19 & 8,24 & 9,43 & 10,21 & 11,16 & 12,49 & 13,50 & 14,15 & 18,47 & 20,56 & 23,53 & 25,55 & 26,52 & 27,51 & 28,45\end{array}$ $\begin{array}{lllllllllllllllllllllllllll}1,16 & 2,23 & 3,25 & 4,24 & 5,27 & 6,47 & 7,15 & 8,51 & 9,45 & 10,46 & 11,55 & 12,48 & 13,17 & 14,49 & 18,50 & 19,53 & 20,54 & 21,44 & 22,52 & 26,56 & 28,43\end{array}$ $\begin{array}{llllllllllllllllllllllll}1,25 & 2,17 & 3,26 & 4,50 & 5,53 & 6,22 & 7,43 & 8,15 & 9,55 & 10,45 & 11,20 & 12,44 & 13,46 & 14,28 & 16,51 & 18,48 & 19,56 & 21,47 & 23,49 & 24,54 & 27,52\end{array}$ $\begin{array}{llllllllllllllllllllllll}1,23 & 2,18 & 3,49 & 4,43 & 5,50 & 6,55 & 7,47 & 8,53 & 9,15 & 10,56 & 11,17 & 12,20 & 13,19 & 14,21 & 16,52 & 22,54 & 24,48 & 25,46 & 26,51 & 27,45 & 28,44\end{array}$ $\begin{array}{llllllllllllllllllllll}1,45 & 2,24 & 3,43 & 4,49 & 5,26 & 6,44 & 7,27 & 8,56 & 9,28 & 10,25 & 11,52 & 12,15 & 13,47 & 14,20 & 16,54 & 17,48 & 18,53 & 19,51 & 21,50 & 22,55 & 23,46\end{array}$ $\begin{array}{lllllllllllllllllllllllllll}1,43 & 2,19 & 3,20 & 4,45 & 5,23 & 6,48 & 7,55 & 8,50 & 9,56 & 10,51 & 11,28 & 12,21 & 13,24 & 14,17 & 15,47 & 16,44 & 18,49 & 22,46 & 25,52 & 26,53 & 27,54\end{array}$

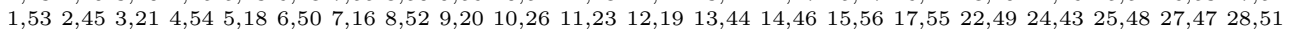

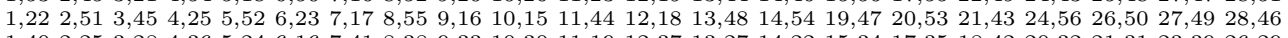
$\begin{array}{lllllllllllllllllllllllllllllll}1,40 & 2,25 & 3,28 & 4,36 & 5,24 & 6,16 & 7,41 & 8,38 & 9,33 & 10,30 & 11,19 & 12,37 & 13,27 & 14,22 & 15,34 & 17,35 & 18,42 & 20,32 & 21,31 & 23,39 & 26,29\end{array}$ $\begin{array}{lllllllllllllllllllllllll}1,36 & 2,28 & 3,39 & 4,32 & 5,17 & 6,19 & 7,29 & 8,33 & 9,18 & 10,27 & 11,41 & 12,24 & 13,20 & 14,30 & 15,37 & 16,35 & 21,42 & 22,31 & 23,38 & 25,34 & 26,40\end{array}$ $\begin{array}{llllllllllllllllllllllll}1,19 & 2,39 & 3,18 & 4,33 & 5,42 & 6,28 & 7,25 & 8,26 & 9,23 & 10,16 & 11,34 & 12,30 & 13,32 & 14,29 & 15,40 & 17,41 & 20,31 & 21,38 & 22,36 & 24,35 & 27,37\end{array}$ $\begin{array}{llllllllllllllllllllllllllllllll}1,24 & 2,34 & 3,37 & 4,28 & 5,15 & 6,39 & 7,42 & 8,27 & 9,32 & 10,29 & 11,18 & 12,40 & 13,22 & 14,23 & 16,30 & 17,38 & 19,36 & 20,41 & 21,35 & 25,31 & 26,33\end{array}$ $\begin{array}{llllllllllllllllllllllll}1,34 & 2,20 & 3,38 & 4,37 & 5,21 & 6,42 & 7,35 & 8,25 & 9,31 & 10,22 & 11,15 & 12,28 & 13,29 & 14,19 & 16,41 & 17,32 & 18,40 & 23,36 & 24,39 & 26,30 & 27,33\end{array}$ $\begin{array}{lllllllllllllllllllll}1,27 & 2,35 & 3,36 & 4,18 & 5,30 & 6,15 & 7,31 & 8,21 & 9,25 & 10,39 & 11,29 & 12,33 & 13,28 & 14,16 & 17,34 & 19,32 & 20,40 & 22,37 & 23,42 & 24,38 & 26,41\end{array}$ $\begin{array}{llllllllllllllllllllllll}1,33 & 2,22 & 3,19 & 4,15 & 5,37 & 6,21 & 7,32 & 8,16 & 9,38 & 10,36 & 11,24 & 12,35 & 13,41 & 14,26 & 17,40 & 18,31 & 20,30 & 23,29 & 25,42 & 27,39 & 28,34\end{array}$

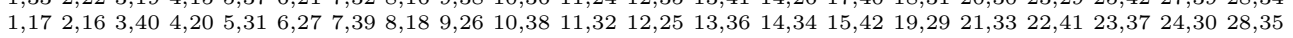
$\begin{array}{llllllllllllllllllllllllllll}1,18 & 2,29 & 3,42 & 4,19 & 5,22 & 6,26 & 7,28 & 8,40 & 9,24 & 10,35 & 11,36 & 12,32 & 13,38 & 14,27 & 15,30 & 16,33 & 17,31 & 20,37 & 21,39 & 23,34 & 25,41\end{array}$

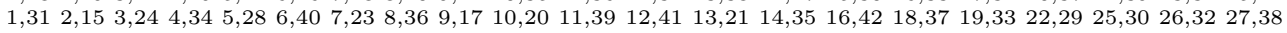
$\begin{array}{llllllllllllllllllllllllll}1,20 & 2,38 & 3,17 & 4,40 & 5,41 & 6,30 & 7,22 & 8,28 & 9,35 & 10,23 & 11,21 & 12,34 & 13,26 & 14,33 & 15,39 & 16,36 & 18,29 & 19,31 & 24,32 & 25,37 & 27,42 \\ 1,15 & 2,27 & 3,33 & 4,30 & 5,19 & 6,29 & 7,26 & 8 & 39 & 9,22 & 10,41 & 11,42 & 12,17 & 13,23 & 14,32 & 16,31 & 18,38 & 20,34 & 21,37 & 24,40 & 25,35 & 28,36\end{array}$

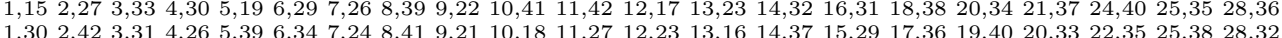

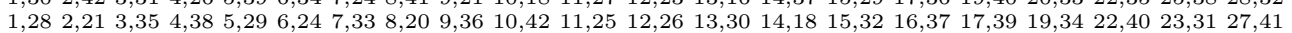

$F_{c}$

$\begin{array}{llllllllllllllllllllllllll}15,40 & 16,33 & 17,36 & 18,44 & 19,31 & 20,52 & 21,50 & 22,51 & 23,53 & 24,30 & 25,42 & 26,41 & 27,54 & 28,43 & 29,48 & 32,55 & 34,46 & 35,47 & 37,45 & 38,49 & 39,56\end{array}$ $\begin{array}{llllllllllllllllllllllllll}15,52 & 16,31 & 17,54 & 18,45 & 19,40 & 20,30 & 21,47 & 22,41 & 23,34 & 24,49 & 25,55 & 26,29 & 27,38 & 28,56 & 32,46 & 33,53 & 35,44 & 36,51 & 37,48 & 39,43 & 42,50\end{array}$ $\begin{array}{lllllllllllllllllllllll}15,42 & 16,51 & 17,35 & 18,43 & 19,32 & 20,46 & 21,37 & 22,52 & 23,31 & 24,39 & 25,49 & 26,33 & 27,45 & 28,53 & 29,56 & 30,50 & 34,48 & 36,44 & 38,54 & 40,47 & 41,55\end{array}$ $\begin{array}{lllllllllllllllllllllllll}15,47 & 16,37 & 17,31 & 18,53 & 19,48 & 20,41 & 21,38 & 22,29 & 23,39 & 24,55 & 25,46 & 26,56 & 27,49 & 28,35 & 30,51 & 32,45 & 33,44 & 34,50 & 36,52 & 40,54 & 42,43\end{array}$ $\begin{array}{lllllllllllllllllllllllllllllll}15,56 & 16,36 & 17,41 & 18,49 & 19,44 & 20,40 & 21,42 & 22,35 & 23,45 & 24,54 & 25,31 & 26,48 & 27,33 & 28,47 & 29,52 & 30,43 & 32,50 & 34,53 & 37,46 & 38,51 & 39,55\end{array}$

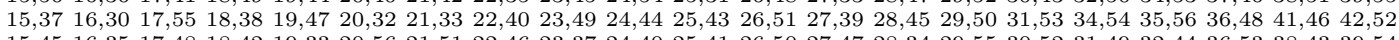

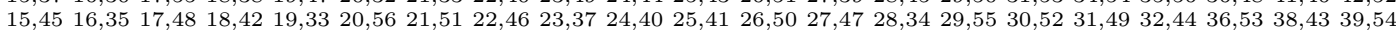
$\begin{array}{lllllllllllllllllllllllllllllllll}15,44 & 16,52 & 17,50 & 18,40 & 19,34 & 20,47 & 21,31 & 22,55 & 23,38 & 24,32 & 25,35 & 26,53 & 27,37 & 28,51 & 29,43 & 30,49 & 33,56 & 36,54 & 39,45 & 41,48 & 42,46\end{array}$ $\begin{array}{llllllllllllllllllllllllllllll}15,46 & 16,53 & 17,39 & 18,37 & 19,56 & 20,34 & 21,35 & 22,54 & 23,29 & 24,38 & 25,52 & 26,55 & 27,51 & 28,32 & 30,48 & 31,50 & 33,47 & 36,43 & 40,45 & 41,49 & 42,44\end{array}$ $\begin{array}{llllllllllllllllllllllllll}15,39 & 16,54 & 17,32 & 18,48 & 19,29 & 20,31 & 21,44 & 22,56 & 23,51 & 24,46 & 25,38 & 26,52 & 27,41 & 28,36 & 30,47 & 33,45 & 34,55 & 35,43 & 37,50 & 40,49 & 42,53\end{array}$ $\begin{array}{lllllllllllllllllllllllll}15,32 & 16,44 & 17,34 & 18,50 & 19,36 & 20,33 & 21,55 & 22,49 & 23,47 & 24,48 & 25,30 & 26,40 & 27,42 & 28,46 & 29,54 & 31,52 & 35,45 & 37,51 & 38,56 & 39,53 & 41,43\end{array}$ $\begin{array}{lllllllllllllllllllllllllllllll}15,29 & 16,42 & 17,38 & 18,47 & 19,53 & 20,37 & 21,43 & 22,31 & 23,36 & 24,35 & 25,48 & 26,44 & 27,46 & 28,49 & 30,54 & 32,56 & 33,55 & 34,45 & 39,50 & 40,52 & 41,51\end{array}$ $\begin{array}{lllllllllllllllllllllllll}15,34 & 16,49 & 17,47 & 18,31 & 19,51 & 20,54 & 21,39 & 22,36 & 23,42 & 24,56 & 25,37 & 26,30 & 27,52 & 28,48 & 29,44 & 32,53 & 33,43 & 35,50 & 38,55 & 40,46 & 41,45\end{array}$ $\begin{array}{lllllllllllllllllllllllllll}15,30 & 16,41 & 17,40 & 18,29 & 19,50 & 20,53 & 21,56 & 22,37 & 23,46 & 24,43 & 25,34 & 26,32 & 27,55 & 28,44 & 31,48 & 33,54 & 35,49 & 36,47 & 38,45 & 39,52 & 42,51\end{array}$

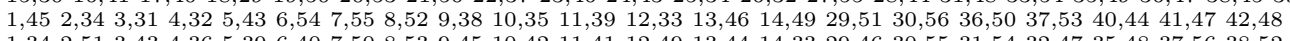

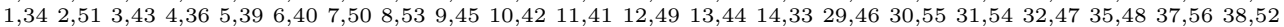
$\begin{array}{lllllllllllllllllllllllll}1,53 & 2,52 & 3,54 & 4,45 & 5,42 & 6,39 & 7,31 & 8,51 & 9,43 & 10,36 & 11,32 & 12,34 & 13,55 & 14,37 & 29,47 & 30,44 & 33,49 & 35,46 & 38,48 & 40,50 & 41,56\end{array}$ $\begin{array}{lllllllllllllllllllllllllll}1,52 & 2,56 & 3,42 & 4,50 & 5,41 & 6,45 & 7,32 & 8,36 & 9,44 & 10,51 & 11,34 & 12,40 & 13,30 & 14,43 & 29,53 & 31,47 & 33,48 & 35,55 & 37,54 & 38,46 & 39,49\end{array}$ $\begin{array}{lllllllllllllllllllllllllll}1,33 & 2,49 & 3,35 & 4,30 & 5,54 & 6,50 & 7,42 & 8,56 & 9,52 & 10,45 & 11,55 & 12,37 & 13,38 & 14,29 & 31,51 & 32,48 & 34,44 & 36,46 & 39,47 & 40,43 & 41,53\end{array}$ $\begin{array}{llllllllllllllllllllllllll}1,30 & 2,45 & 3,56 & 4,33 & 5,50 & 6,48 & 7,39 & 8,40 & 9,35 & 10,46 & 11,29 & 12,41 & 13,53 & 14,51 & 31,44 & 32,49 & 34,43 & 36,55 & 37,52 & 38,47 & 42,54\end{array}$ $\begin{array}{lllllllllllllllllllll}1,30 & 2,45 & 3,56 & 4,33 & 5,50 & 6,48 & 7,39 & 8,40 & 9,35 & 10,46 & 11,29 & 12,41 & 13,53 & 14,51 & 31,44 & 32,49 & 34,43 & 36,55 & 37,52 & 38,47 & 42,54 \\ 1,40 & 2,43 & 3,36 & 4,38 & 5,31 & 6,42 & 7,47 & 8,39 & 9,32 & 10,53 & 11,50 & 12,44 & 13,48 & 14,46 & 29,49 & 30,45 & 33,52 & 34,56 & 35,51 & 37,55 & 41,54\end{array}$ $\begin{array}{llllllllllllllllllllll}1,40 & 2,43 & 3,36 & 4,38 & 5,31 & 6,42 & 7,47 & 8,39 & 9,32 & 10,53 & 11,50 & 12,44 & 13,48 & 14,46 & 29,49 & 30,45 & 33,52 & 34,56 & 35,51 & 37,55 & 41,54 \\ 1,31 & 2,38 & 3,40 & 4,48 & 5,52 & 6,55 & 7,43 & 8,47 & 9,51 & 10,41 & 11,36 & 12,45 & 13,29 & 14,30 & 32,54 & 33,50 & 34,49 & 35,53 & 37,44 & 39,46 & 42,56\end{array}$ $\begin{array}{lllllllllllllllllllll}1,31 & 2,38 & 3,40 & 4,48 & 5,52 & 6,55 & 7,43 & 8,47 & 9,51 & 10,41 & 11,36 & 12,45 & 13,29 & 14,30 & 32,54 & 33,50 & 34,49 & 35,53 & 37,44 & 39,46 & 42,56 \\ 1,54 & 2,46 & 3,45 & 4,43 & 5,30 & 6,34 & 7,35 & 8,50 & 9,33 & 10,29 & 11,52 & 12,32 & 13,36 & 14,48 & 31,56 & 37,47 & 38,53 & 39,51 & 40,55 & 41,44 & 42,49\end{array}$

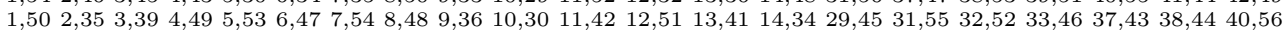
$\begin{array}{llllllllllllllllllllllllll}1,43 & 2,29 & 3,49 & 4,40 & 5,37 & 6,46 & 7,56 & 8,33 & 9,31 & 10,38 & 11,44 & 12,55 & 13,50 & 14,32 & 30,53 & 34,51 & 35,54 & 36,45 & 39,48 & 41,52 & 42,47\end{array}$ $\begin{array}{lllllllllllllllllllllll}1,47 & 2,42 & 3,37 & 4,34 & 5,45 & 6,29 & 7,33 & 8,38 & 9,55 & 10,56 & 11,54 & 12,48 & 13,32 & 14,53 & 30,46 & 31,43 & 35,52 & 36,49 & 39,44 & 40,51 & 41,50\end{array}$ $\begin{array}{llllllllllllllllllllllllll}1,44 & 2,39 & 3,33 & 4,37 & 5,48 & 6,30 & 7,29 & 8,41 & 9,46 & 10,49 & 11,43 & 12,35 & 13,47 & 14,54 & 31,45 & 32,51 & 34,52 & 36,56 & 38,50 & 40,53 & 42,55\end{array}$ $\begin{array}{llllllllllllllllllllll}1,36 & 2,50 & 3,38 & 4,54 & 5,29 & 6,44 & 7,41 & 8,55 & 9,56 & 10,39 & 11,53 & 12,30 & 13,52 & 14,35 & 31,46 & 32,43 & 33,51 & 34,47 & 37,49 & 40,48 & 42,45\end{array}$ $\begin{array}{llllllllllllllllllllll}1,15 & 2,54 & 3,52 & 4,46 & 5,21 & 6,51 & 7,25 & 8,18 & 9,50 & 10,23 & 11,48 & 12,43 & 13,27 & 14,26 & 16,56 & 17,44 & 19,45 & 20,49 & 22,53 & 24,47 & 28,55\end{array}$ $\begin{array}{lllllllllllllllllllllll}1,20 & 2,53 & 3,51 & 4,18 & 5,22 & 6,56 & 7,26 & 8,44 & 9,48 & 10,27 & 11,25 & 12,47 & 13,16 & 14,52 & 15,54 & 17,46 & 19,55 & 21,49 & 23,43 & 24,45 & 28,50\end{array}$ $\begin{array}{llllllllllllllllllllll}1,49 & 2,55 & 3,19 & 4,26 & 5,44 & 6,27 & 7,45 & 8,46 & 9,54 & 10,20 & 11,21 & 12,53 & 13,28 & 14,18 & 15,51 & 16,47 & 17,43 & 22,48 & 23,56 & 24,52 & 25,50\end{array}$

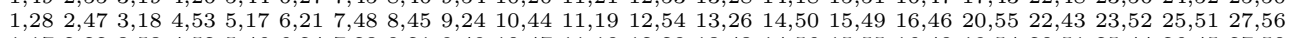

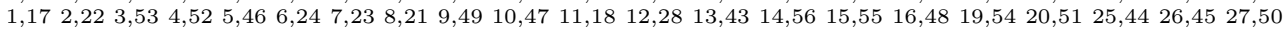
$\begin{array}{lllllllllllllllllllllllll}1,24 & 2,48 & 3,17 & 4,15 & 5,55 & 6,19 & 7,44 & 8,26 & 9,22 & 10,43 & 11,46 & 12,56 & 13,49 & 14,27 & 16,45 & 18,51 & 20,50 & 21,53 & 23,54 & 25,47 & 28,52\end{array}$ $\begin{array}{llllllllllllllllllllll}1,55 & 2,15 & 3,44 & 4,20 & 5,47 & 6,49 & 7,51 & 8,28 & 9,23 & 10,48 & 11,27 & 12,52 & 13,22 & 14,19 & 16,43 & 17,56 & 18,46 & 21,45 & 24,50 & 25,53 & 26,54\end{array}$ $\begin{array}{lllllllllllllllllllllllll}1,51 & 2,21 & 3,48 & 4,5 & 5,28 & 6,15 & 7,24 & 8,54 & 9,53 & 10,16 & 11,56 & 12,25 & 13,23 & 14,47 & 17,45 & 18,52 & 19,49 & 20,43 & 22,50 & 26,46 & 27,44\end{array}$ $\begin{array}{llllllllllllllllllllllllll}1,48 & 2,27 & 3,46 & 4,19 & 5,51 & 6,28 & 7,52 & 8,49 & 9,47 & 10,22 & 11,45 & 12,24 & 13,21 & 14,23 & 15,50 & 16,55 & 17,53 & 18,54 & 20,44 & 25,56 & 26,43\end{array}$ $\begin{array}{llllllllllllllllllllll}1,56 & 2,28 & 3,50 & 4,47 & 5,19 & 6,16 & 7,53 & 8,20 & 9,26 & 10,52 & 11,24 & 12,23 & 13,51 & 14,44 & 15,48 & 17,49 & 18,55 & 21,46 & 22,45 & 25,54 & 27,43\end{array}$ $\begin{array}{lllllllllllllllllllllll}1,19 & 2,20 & 3,28 & 4,44 & 5,56 & 6,26 & 7,49 & 8,27 & 9,25 & 10,18 & 11,51 & 12,46 & 13,54 & 14,45 & 15,43 & 16,50 & 17,52 & 21,48 & 22,47 & 23,55 & 24,53\end{array}$ $\begin{array}{llllllllllllllllllllllllllll}1,18 & 2,25 & 3,55 & 4,28 & 5,49 & 6,53 & 7,46 & 8,43 & 9,21 & 10,54 & 11,15 & 12,17 & 13,56 & 14,16 & 19,52 & 20,45 & 22,44 & 23,50 & 24,51 & 26,47 & 27,48\end{array}$ $\begin{array}{lllllllllllllllllllllllll}1,46 & 2,16 & 3,24 & 4,56 & 5,15 & 6,52 & 7,28 & 8,25 & 9,18 & 10,55 & 11,47 & 12,50 & 13,45 & 14,22 & 17,51 & 19,43 & 20,48 & 21,54 & 23,44 & 26,49 & 27,53\end{array}$ $\begin{array}{lllllllllllllllllllllllllll}1,27 & 2,44 & 3,47 & 4,51 & 5,24 & 6,43 & 7,22 & 8,16 & 9,17 & 10,50 & 11,49 & 12,26 & 13,20 & 14,55 & 15,53 & 18,56 & 19,46 & 21,52 & 23,48 & 25,45 & 28,54\end{array}$ $\begin{array}{llllllllllllllllllllllll}1,23 & 2,24 & 3,34 & 4,42 & 5,35 & 6,36 & 7,18 & 8,31 & 9,30 & 10,17 & 11,20 & 12,27 & 13,37 & 14,15 & 16,38 & 19,41 & 21,40 & 22,32 & 25,33 & 26,39 & 28,29\end{array}$ $\begin{array}{llllllllllllllllllllllllll}1,29 & 2,17 & 3,16 & 4,31 & 5,38 & 6,18 & 7,15 & 8,37 & 9,28 & 10,26 & 11,33 & 12,22 & 13,35 & 14,39 & 19,42 & 20,36 & 21,34 & 23,32 & 24,41 & 25,40 & 27,30\end{array}$ $\begin{array}{llllllllllllllllllllllll}1,38 & 2,37 & 3,26 & 4,22 & 5,32 & 6,25 & 7,40 & 8,29 & 9,19 & 10,34 & 11,28 & 12,18 & 13,31 & 14,24 & 15,36 & 16,39 & 17,33 & 20,42 & 21,30 & 23,41 & 27,35\end{array}$ $\begin{array}{lllllllllllllllllllll}1,26 & 2,36 & 3,25 & 4,41 & 5,33 & 6,37 & 7,16 & 8,17 & 9,34 & 10,15 & 11,31 & 12,20 & 13,18 & 14,38 & 19,30 & 21,32 & 22,39 & 23,35 & 24,42 & 27,29 & 28,40\end{array}$

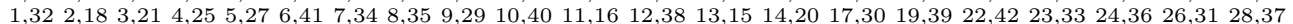

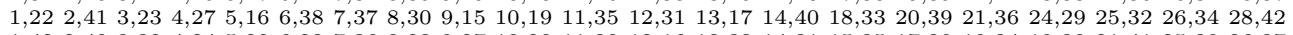

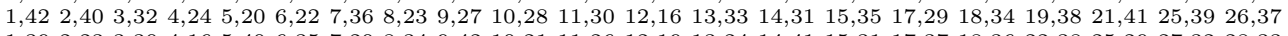

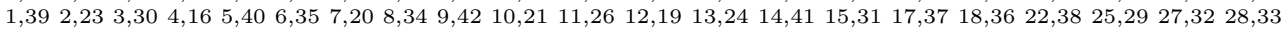
$\begin{array}{llllllllllllllllllllllllll}1,21 & 2,32 & 3,41 & 4,17 & 5,25 & 6,33 & 7,38 & 8,24 & 9,20 & 10,31 & 11,40 & 12,15 & 13,39 & 14,28 & 16,29 & 18,35 & 19,37 & 22,34 & 23,30 & 26,42 & 27,36\end{array}$ $\begin{array}{llllllllllllllllllllllllllll}1,41 & 2,30 & 3,22 & 4,29 & 5,23 & 6,20 & 7,21 & 8,42 & 9,16 & 10,32 & 11,17 & 12,36 & 13,40 & 14,25 & 15,33 & 18,39 & 19,35 & 24,37 & 26,38 & 27,34 & 28,31\end{array}$ $\begin{array}{lllllllllllllllllllllllllll}1,16 & 2,33 & 3,27 & 4,39 & 5,34 & 6,31 & 7,30 & 8,15 & 9,37 & 10,24 & 11,22 & 12,42 & 13,19 & 14,17 & 18,32 & 20,38 & 21,29 & 23,40 & 25,36 & 26,35 & 28,41\end{array}$ $\begin{array}{llllllllllllllllllllllllllllllll}1,25 & 2,19 & 3,29 & 4,23 & 5,18 & 6,17 & 7,27 & 8,32 & 9,40 & 10,37 & 11,38 & 12,39 & 13,42 & 14,21 & 15,41 & 16,34 & 20,35 & 22,33 & 24,31 & 26,36 & 28,30\end{array}$ $\begin{array}{lllllllllllllllllllll}1,35 & 2,31 & 3,20 & 4,21 & 5,26 & 6,23 & 7,19 & 8,22 & 9,41 & 10,33 & 11,37 & 12,29 & 13,25 & 14,36 & 15,38 & 16,32 & 17,42 & 18,30 & 24,34 & 27,40 & 28,39\end{array}$

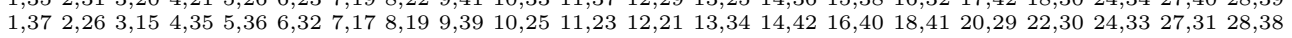




\section{A Room frame of type $2^{19} 18^{1}$}

$F_{r}$

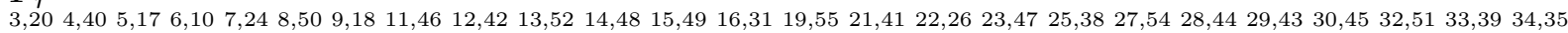
$36,53 \quad 37,56$

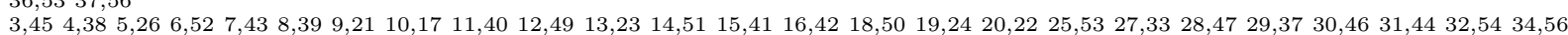
$35,4836,55$

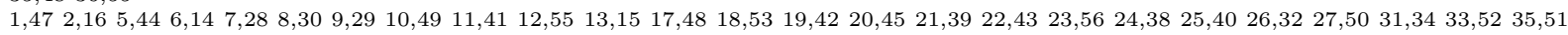
$36,5437,46$

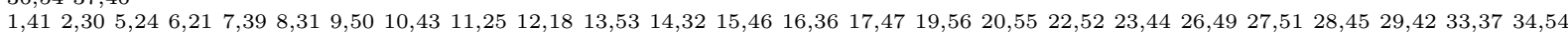
$35,40 \quad 38,48$

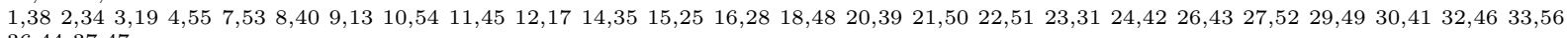
$36,4437,47$

$\begin{array}{lllllllllllllllllllllllll}1,18 & 2,9 & 3,49 & 4,11 & 7,51 & 8,48 & 10,56 & 12,33 & 13,29 & 14,47 & 15,27 & 16,40 & 17,52 & 19,45 & 20,21 & 22,34 & 23,46 & 24,32 & 25,54 & 26,55 & 28,41 & 30,39 & 31,50 & 35,53 & 36,42\end{array}$ $37,4338,44$

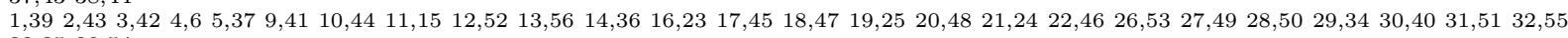
$33,3538,54$

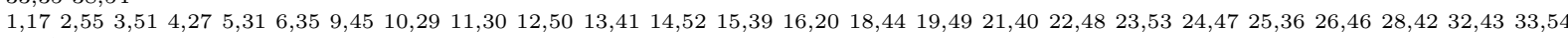
$34,3738,56$

$\begin{array}{lllllllllllllllllllllllllll}1,50 & 2,39 & 3,56 & 4,44 & 5,54 & 6,28 & 7,26 & 8,25 & 11,23 & 12,45 & 13,51 & 14,19 & 15,40 & 16,29 & 17,53 & 18,31 & 20,52 & 21,43 & 22,47 & 24,46 & 27,42 & 30,38 & 32,35 & 33,55 & 34,49\end{array}$ $36,4837,41$

$\begin{array}{llllllllllllllllllllllllll}1,44 & 2,21 & 3,18 & 4,26 & 5,36 & 6,24 & 7,29 & 8,28 & 11,51 & 12,48 & 13,27 & 14,56 & 15,45 & 16,43 & 17,41 & 19,40 & 20,30 & 22,54 & 23,42 & 25,39 & 31,46 & 32,50 & 33,53 & 34,52 & 35,47\end{array}$ $37,4938,55$

$\begin{array}{lllllllllllllllllllllllll}1,46 & 2,40 & 3,35 & 4,43 & 5,7 & 6,44 & 8,42 & 9,19 & 10,45 & 13,33 & 14,53 & 15,56 & 16,41 & 17,55 & 18,32 & 20,28 & 21,47 & 22,29 & 23,48 & 24,27 & 25,49 & 26,50 & 30,34 & 31,54 & 36,52\end{array}$ $37,5138,39$

$\begin{array}{llllllllllllllllllllllllllllll}1,20 & 2,46 & 3,54 & 4,34 & 5,53 & 6,55 & 7,56 & 8,16 & 9,51 & 10,50 & 13,28 & 14,27 & 15,26 & 17,30 & 18,45 & 19,47 & 21,36 & 22,41 & 23,38 & 24,49 & 25,42 & 29,52 & 31,48 & 32,40 & 33,44\end{array}$ $35,4337,39$

$\begin{array}{lllllllllllllllllllllllllllll}1,34 & 2,36 & 3,46 & 4,24 & 5,43 & 6,27 & 7,32 & 8,55 & 9,42 & 10,20 & 11,44 & 12,39 & 15,47 & 16,38 & 17,56 & 18,51 & 19,22 & 21,53 & 23,52 & 25,45 & 26,40 & 28,48 & 29,54 & 30,37 & 31,49\end{array}$ $33,5035,41$

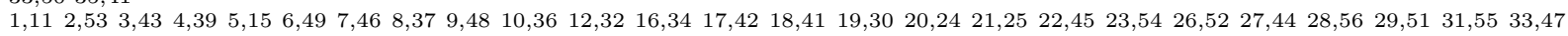
$35,5038,40$

$\begin{array}{lllllllllllllllllllllllllll}1,43 & 2,35 & 3,44 & 4,29 & 5,10 & 6,46 & 7,9 & 8,27 & 11,39 & 12,53 & 13,42 & 14,33 & 17,19 & 18,54 & 20,50 & 21,56 & 22,55 & 23,40 & 24,36 & 25,47 & 26,31 & 28,52 & 30,51 & 32,45 & 34,41\end{array}$ $37,4838,49$

$\begin{array}{lllllllllllllllllllllllllllllll}1,45 & 2,44 & 3,25 & 4,51 & 5,42 & 6,41 & 7,55 & 8,32 & 9,46 & 10,33 & 11,36 & 12,19 & 13,31 & 14,30 & 17,39 & 18,21 & 20,43 & 22,23 & 24,48 & 26,56 & 27,40 & 28,49 & 29,53 & 34,47 & 35,54\end{array}$ $37,5238,50$

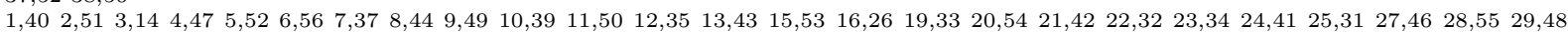
$30,3638,45$

$\begin{array}{llllllllllllllllllllllllllllll}1,7 & 2,49 & 3,11 & 4,53 & 5,51 & 6,50 & 8,20 & 9,43 & 10,47 & 12,40 & 13,32 & 14,31 & 15,55 & 16,39 & 19,46 & 21,38 & 22,35 & 23,27 & 24,52 & 25,37 & 26,48 & 28,54 & 29,45 & 30,42 & 33,41\end{array}$

$\begin{array}{llllllllllllllllllllllllllll}34,44 & 36,56 & 1,26 & 2,41 & 3,48 & 4,49 & 5,55 & 6,30 & 7,52 & 8,56 & 9,53 & 10,37 & 11,38 & 12,22 & 13,54 & 14,39 & 15,28 & 16,46 & 17,34 & 18,43 & 21,44 & 23,51 & 24,35 & 25,50 & 27,47 & 29,36 & 31,40\end{array}$ $1,262,413,4$

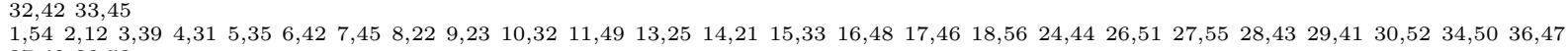

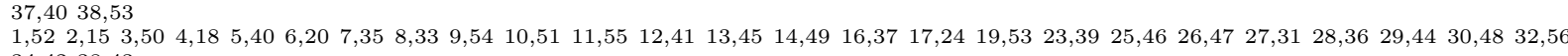
$34,4238,43$

$\begin{array}{lllllllllllllllllllllllllll}1,42 & 2,14 & 3,36 & 4,56 & 5,39 & 6,29 & 7,41 & 8,49 & 9,24 & 10,23 & 11,52 & 12,46 & 13,38 & 15,50 & 16,55 & 17,27 & 18,28 & 19,51 & 20,40 & 25,44 & 26,33 & 30,43 & 31,47 & 32,48 & 34,53\end{array}$ $35,4537,54$

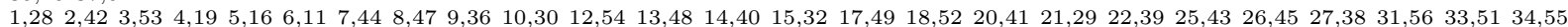
$35,4637,50$

$\begin{array}{lllllllllllllllllllllllllllll}1,13 & 2,4 & 3,40 & 5,21 & 6,12 & 7,42 & 8,34 & 9,52 & 10,55 & 11,53 & 14,44 & 15,48 & 16,47 & 17,20 & 18,39 & 19,54 & 22,30 & 25,56 & 26,41 & 27,32 & 28,33 & 29,50 & 31,43 & 35,49 & 36,51\end{array}$ $37,4538,46$

$\begin{array}{llllllllllllllllllllllllllll}1,16 & 2,52 & 3,38 & 4,17 & 5,56 & 6,53 & 7,14 & 8,45 & 9,27 & 10,48 & 11,42 & 12,29 & 13,46 & 15,44 & 18,23 & 19,50 & 20,47 & 21,54 & 22,49 & 24,43 & 28,39 & 30,35 & 31,41 & 32,33 & 34,51\end{array}$ $36,4037,55$

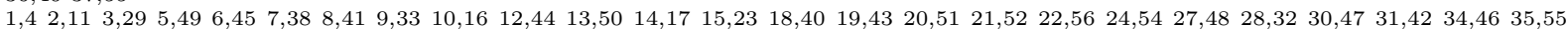
$36,3937,53$

$\begin{array}{llllllllllllllllllllllllll}1,56 & 2,47 & 3,13 & 4,52 & 5,50 & 6,19 & 7,49 & 8,51 & 9,16 & 10,12 & 11,48 & 14,26 & 15,18 & 17,32 & 20,23 & 21,45 & 22,33 & 24,55 & 25,41 & 29,40 & 30,54 & 31,53 & 34,43 & 35,39 & 36,46\end{array}$ $37,4438,42$

$\begin{array}{llllllllllllllllllllllllllllllllll}1,3 & 2,50 & 4,54 & 5,19 & 6,39 & 7,17 & 8,35 & 9,55 & 10,21 & 11,31 & 12,26 & 13,47 & 14,42 & 15,43 & 16,45 & 18,38 & 20,53 & 22,37 & 23,49 & 24,40 & 25,51 & 29,56 & 30,44 & 32,52 & 33,46\end{array}$ $34,4836,41$

$\begin{array}{lllllllllllllllllllllllllllllll}1,55 & 2,25 & 3,47 & 4,46 & 5,27 & 6,51 & 7,12 & 8,26 & 9,44 & 10,28 & 11,54 & 13,18 & 14,50 & 15,22 & 16,56 & 17,40 & 19,37 & 20,31 & 21,48 & 23,41 & 24,53 & 32,39 & 33,43 & 34,45 & 35,42\end{array}$ $36,4938,52$

$\begin{array}{llllllllllllllllllllllllll}1,9 & 2,48 & 3,8 & 4,41 & 5,34 & 6,17 & 7,47 & 10,52 & 11,43 & 12,25 & 13,36 & 14,46 & 15,35 & 16,51 & 18,42 & 19,44 & 20,56 & 21,55 & 22,50 & 23,37 & 24,45 & 26,54 & 27,53 & 28,40 & 31,39\end{array}$ $32,4933,38$

$\begin{array}{llllllllllllllllllllllllllllllll}1,5 & 2,45 & 3,28 & 4,50 & 6,48 & 7,40 & 8,53 & 9,56 & 10,41 & 11,13 & 12,37 & 14,54 & 15,34 & 16,44 & 17,51 & 18,55 & 19,52 & 20,46 & 21,26 & 22,38 & 23,35 & 24,25 & 27,39 & 29,47 & 30,49\end{array}$

$\begin{array}{lllllllllllllllllllllllllllll}33,42 & 36,43 & 1,30 & 2,3 & 4,28 & 5,22 & 6,34 & 7,48 & 8,38 & 9,39 & 10,53 & 11,47 & 12,56 & 13,17 & 14,45 & 15,54 & 16,52 & 18,27 & 19,41 & 20,49 & 21,51 & 23,43 & 24,26 & 25,55 & 29,46 & 33,40 & 35,44\end{array}$ $1,302,34,28$

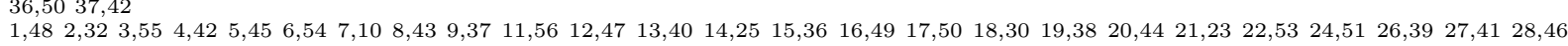
$29,3135,52$

$\begin{array}{llllllllllllllllllllllllllllllllll}1,49 & 2,37 & 3,26 & 4,48 & 5,41 & 6,8 & 7,50 & 9,25 & 10,40 & 11,19 & 12,20 & 13,39 & 14,43 & 15,42 & 16,53 & 17,54 & 18,22 & 21,46 & 23,45 & 24,56 & 27,36 & 28,51 & 29,35 & 30,55 & 31,52\end{array}$ $32,44 \quad 38,47$

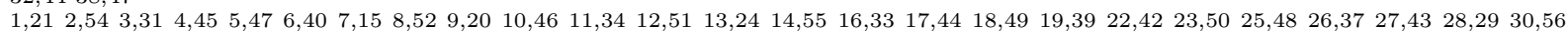
$32,53 \quad 38,41$

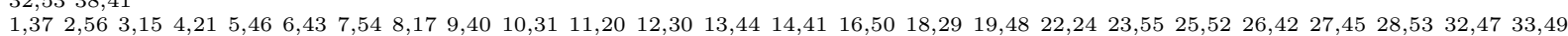
$34,3938,51$

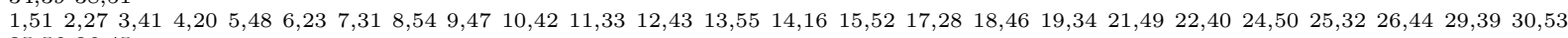
$35,5636,45$

$\begin{array}{llllllllllllllllllllllllllllllllll}1,53 & 2,19 & 3,52 & 4,35 & 5,9 & 6,47 & 7,21 & 8,46 & 10,25 & 11,28 & 12,23 & 13,49 & 14,18 & 15,51 & 16,54 & 17,43 & 20,42 & 22,44 & 24,39 & 26,36 & 27,56 & 29,55 & 30,50 & 31,45 & 32,41\end{array}$ $33,4834,40$

$\begin{array}{lllllllllllllllllllllllll}1,8 & 2,17 & 3,5 & 4,13 & 6,16 & 7,33 & 9,30 & 10,35 & 11,18 & 12,38 & 14,15 & 19,36 & 20,26 & 21,32 & 22,27 & 23,29 & 24,34 & 25,28 & 31,37\end{array}$ $\begin{array}{lllllllllllllllllllllllll}1,10 & 2,5 & 3,12 & 4,32 & 6,25 & 7,11 & 8,36 & 9,22 & 13,30 & 14,38 & 15,17 & 16,27 & 18,35 & 19,23 & 20,37 & 21,34 & 24,33 & 26,29 & 28,31\end{array}$ $\begin{array}{lllllllllllllllllllllllll}1,25 & 2,24 & 3,6 & 4,15 & 5,30 & 7,36 & 8,23 & 9,28 & 10,34 & 11,26 & 12,27 & 13,22 & 14,37 & 16,32 & 17,21 & 18,20 & 19,29 & 31,33 & 35,38\end{array}$

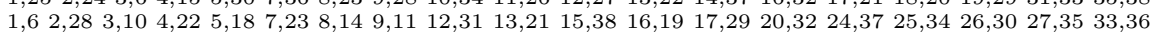
$\begin{array}{llllllllllllllllllll}1,6 & 2,28 & 3,10 & 4,22 & 5,18 & 7,23 & 8,14 & 9,11 & 12,31 & 13,21 & 15,38 & 16,19 & 17,29 & 20,32 & 24,37 & 25,34 & 26,30 & 27,35 & 33,36 \\ 1,24 & 2,22 & 3,32 & 4,37 & 5,14 & 6,31 & 7,25 & 8,19 & 9,35 & 10,15 & 11,21 & 12,28 & 13,16 & 17,38 & 18,26 & 20,33 & 23,30 & 27,29 & 34,36\end{array}$

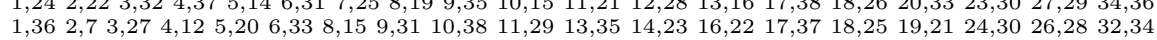
$\begin{array}{lllllllllllllllllllll}1,32 & 2,23 & 3,9 & 4,33 & 5,29 & 6,26 & 7,19 & 8,18 & 10,13 & 11,24 & 12,16 & 14,28 & 15,37 & 17,31 & 20,35 & 21,30 & 22,25 & 27,34 & 36,38\end{array}$

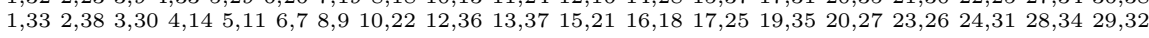




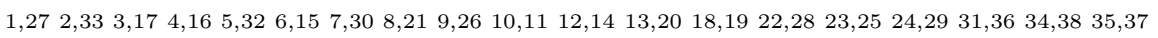
$\begin{array}{llllllllllllllllllllllllllll}1,29 & 2,10 & 3,7 & 4,8 & 5,33 & 6,38 & 9,14 & 11,16 & 12,24 & 13,34 & 15,30 & 17,26 & 18,36 & 19,32 & 20,25 & 21,35 & 22,31 & 23,28 & 27,37\end{array}$ $\begin{array}{llllllllllllllllllllll}1,12 & 2,8 & 3,21 & 4,23 & 5,13 & 6,22 & 7,18 & 9,34 & 10,27 & 11,37 & 14,29 & 15,24 & 16,25 & 17,33 & 19,28 & 20,36 & 26,35 & 30,31 & 32,38\end{array}$ $\begin{array}{lllllllllllllllllllllllll}1,19 & 2,20 & 3,34 & 4,10 & 5,28 & 6,13 & 7,16 & 8,12 & 9,15 & 11,32 & 14,24 & 17,35 & 18,37 & 21,31 & 22,36 & 23,33 & 25,30 & 26,27 & 29,38\end{array}$

$\begin{array}{llllllllllllllllllllll}1,22 & 2,6 & 3,23 & 4,7 & 5,12 & 8,11 & 9,38 & 10,26 & 13,19 & 14,20 & 15,31 & 16,24 & 17,36 & 18,34 & 21,37 & 25,27 & 28,35 & 29,33 & 30,32 \\ 1,35 & 2,13 & 3,37 & 4,36 & 5,23 & 6,32 & 7,27 & 8,29 & 9,17 & 10,14 & 11,22 & 12,15 & 16,30 & 18,24 & 19,31 & 20,34 & 21,28 & 25,33 & 26,38\end{array}$

$\begin{array}{llllllllllllllllllllllll}1,35 & 2,13 & 3,37 & 4,36 & 5,23 & 6,32 & 7,27 & 8,29 & 9,17 & 10,14 & 11,22 & 12,15 & 16,30 & 18,24 & 19,31 & 20,34 & 21,28 & 25,33 & 26,38 \\ 1,14 & 2,26 & 3,22 & 4,5 & 6,9 & 7,34 & 8,24 & 10,18 & 11,27 & 12,13 & 15,19 & 16,21 & 17,23 & 20,29 & 25,35 & 28,37 & 30,33 & 31,38 & 32,36\end{array}$

$\begin{array}{llllllllllllllllllll}1,14 & 2,26 & 3,22 & 4,5 & 6,9 & 7,34 & 8,24 & 10,18 & 11,27 & 12,13 & 15,19 & 16,21 & 17,23 & 20,29 & 25,35 & 28,37 & 30,33 & 31,38 & 32,36 \\ 1,15 & 2,18 & 3,16 & 4,30 & 5,38 & 6,37 & 7,20 & 8,10 & 9,32 & 11,17 & 12,34 & 13,26 & 14,22 & 19,27 & 21,33 & 23,36 & 24,28 & 25,29 & 31,35\end{array}$

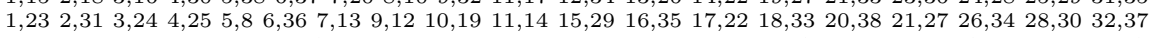

$\begin{array}{lllllllllllllllllllllllll}1,31 & 2,29 & 3,33 & 4,9 & 5,25 & 6,18 & 7,22 & 8,13 & 10,24 & 11,35 & 12,21 & 14,34 & 15,20 & 16,17 & 19,26 & 23,32 & 27,30 & 28,38 & 36,37\end{array}$

$F_{c}$

$\begin{array}{lllllllllllllllllllllllll}3,39 & 4,13 & 5,20 & 6,9 & 7,44 & 8,29 & 10,42 & 11,26 & 12,31 & 14,43 & 15,55 & 16,24 & 17,41 & 18,47 & 19,35 & 21,48 & 22,53 & 23,36 & 25,50 & 27,52 & 28,45 & 30,49 & 32,56 & 33,40 & 34,51\end{array}$ $37,5438,46$

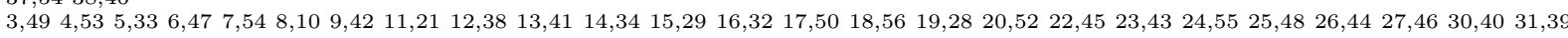
$35,3736,51$

$\begin{array}{llllllllllllllllllllllllllll}1,39 & 2,45 & 5,30 & 6,37 & 7,55 & 8,13 & 9,38 & 10,47 & 11,27 & 12,54 & 14,41 & 15,43 & 16,51 & 17,26 & 18,40 & 19,50 & 20,35 & 21,46 & 22,42 & 23,29 & 24,44 & 25,56 & 28,52 & 31,48 & 32,34\end{array}$ $33,5336,49$

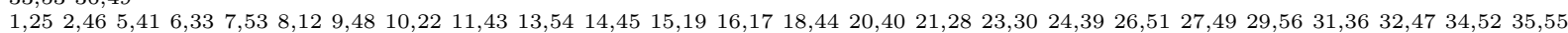
$37,5038,42$

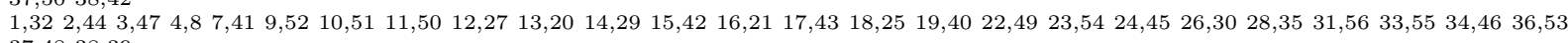
$37,4838,39$

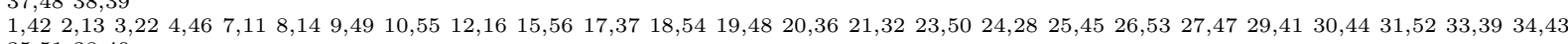
$35,5138,40$

$\begin{array}{lllllllllllllllllllllllll}1,33 & 2,22 & 3,16 & 4,44 & 5,28 & 6,45 & 9,53 & 10,35 & 11,42 & 12,55 & 13,30 & 14,50 & 15,52 & 17,51 & 18,41 & 19,21 & 20,32 & 23,48 & 24,49 & 25,54 & 26,38 & 27,40 & 29,46 & 31,43 & 34,39\end{array}$ $36,4737,56$

$\begin{array}{lllllllllllllllllllllllllllll}1,31 & 2,10 & 3,50 & 4,43 & 5,46 & 6,25 & 9,51 & 11,22 & 12,44 & 13,40 & 14,39 & 15,37 & 16,56 & 17,48 & 18,19 & 20,41 & 21,53 & 23,33 & 24,30 & 26,54 & 27,55 & 28,47 & 29,42 & 32,52 & 34,49\end{array}$ $35,3836,45$

$\begin{array}{lllllllllllllllllllllllll}1,46 & 2,51 & 3,32 & 4,52 & 5,49 & 6,53 & 7,13 & 8,50 & 11,44 & 12,43 & 14,22 & 15,39 & 16,27 & 17,31 & 18,20 & 19,26 & 21,55 & 23,56 & 24,48 & 25,47 & 28,54 & 29,38 & 30,41 & 33,36 & 34,42\end{array}$ $35,4037,45$

$\begin{array}{llllllllllllllllllllllllllllllll}1,24 & 2,18 & 3,52 & 4,47 & 5,25 & 6,13 & 7,45 & 8,55 & 11,54 & 12,14 & 15,30 & 16,40 & 17,23 & 19,43 & 20,53 & 21,34 & 22,27 & 26,48 & 28,39 & 29,50 & 31,44 & 32,49 & 33,42 & 35,56 & 36,46\end{array}$ $37,5138,41$

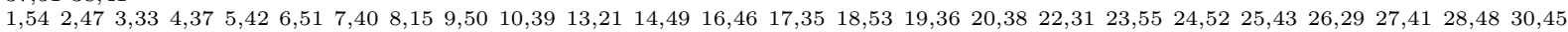
$32,4434,56$

$\begin{array}{llllllllllllllllllllllllllllllllll}1,19 & 2,52 & 3,7 & 4,41 & 5,47 & 6,22 & 8,18 & 9,46 & 10,54 & 13,53 & 14,48 & 15,40 & 16,43 & 17,36 & 20,27 & 21,42 & 23,32 & 24,37 & 25,28 & 26,39 & 29,51 & 30,50 & 31,49 & 33,45 & 34,55\end{array}$ $35,4438,56$

$\begin{array}{llllllllllllllllllllllllllllllll}1,50 & 2,7 & 3,42 & 4,15 & 5,51 & 6,52 & 8,45 & 9,44 & 10,41 & 11,24 & 12,47 & 16,18 & 17,29 & 19,54 & 20,56 & 21,27 & 22,39 & 23,53 & 25,46 & 26,43 & 28,55 & 30,32 & 31,35 & 33,49 & 34,40\end{array}$ $36,3738,48$

$\begin{array}{lllllllllllllllllllllllll}1,53 & 2,24 & 3,12 & 4,7 & 5,8 & 6,32 & 9,15 & 10,50 & 11,29 & 16,44 & 17,52 & 18,39 & 19,51 & 20,33 & 21,41 & 22,55 & 23,45 & 25,40 & 26,46 & 27,34 & 28,49 & 30,56 & 31,54 & 35,47 & 36,48\end{array}$ $37,42 \quad 38,43$

$\begin{array}{lllllllllllllllllllllllll}1,51 & 2,6 & 3,37 & 4,22 & 5,40 & 7,18 & 8,19 & 9,56 & 10,43 & 11,48 & 12,39 & 13,49 & 14,55 & 17,46 & 20,25 & 21,31 & 23,26 & 24,41 & 27,45 & 28,50 & 29,52 & 30,42 & 32,54 & 33,47 & 34,38\end{array}$ $35,5336,44$

$\begin{array}{llllllllllllllllllllllllllll}1,52 & 2,54 & 3,27 & 4,9 & 5,48 & 6,39 & 7,20 & 8,11 & 10,49 & 12,36 & 13,34 & 14,38 & 17,22 & 18,42 & 19,56 & 21,50 & 23,41 & 24,51 & 25,33 & 26,40 & 28,53 & 29,45 & 30,47 & 31,55 & 32,43\end{array}$ $35,4637,44$

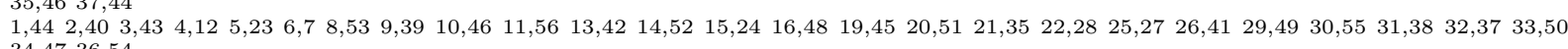
$\begin{array}{llllllllllllllllllllllllllllllllllllllllll}34,47 & 36,54 \\ 1,6 & 2,55 & 3,24 & 4,33 & 5,38 & 7,25 & 8,9 & 10,53 & 11,49 & 12,21 & 13,46 & 14,37 & 15,41 & 16,50 & 19,52 & 20,42 & 22,56 & 23,40 & 26,45 & 27,44 & 28,51 & 29,43 & 30,39 & 31,47 & 32,36\end{array}$

$34,4835,54$

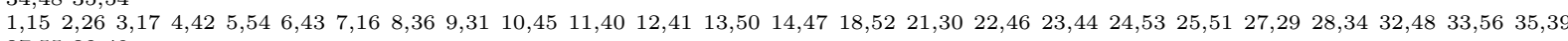
$37,5538,49$

$\begin{array}{llllllllllllllllllllllllllllllllll}1,14 & 2,23 & 3,40 & 4,36 & 5,32 & 6,16 & 7,49 & 8,47 & 9,12 & 10,44 & 11,39 & 13,26 & 15,53 & 17,21 & 18,50 & 22,41 & 24,42 & 25,55 & 27,43 & 28,56 & 29,54 & 30,31 & 33,48 & 34,45 & 35,52\end{array}$ $37,4638,51$

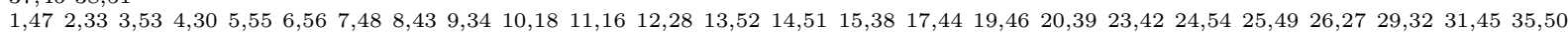
$36,4137,40$

$\begin{array}{lllllllllllllllllllllllllllllllllll}1,56 & 2,20 & 3,23 & 4,49 & 5,43 & 6,40 & 7,30 & 8,41 & 9,35 & 10,24 & 11,47 & 12,45 & 13,44 & 14,42 & 15,51 & 16,39 & 17,54 & 18,55 & 19,27 & 25,53 & 26,34 & 28,37 & 29,48 & 31,50 & 32,46\end{array}$ $33,5236,38$

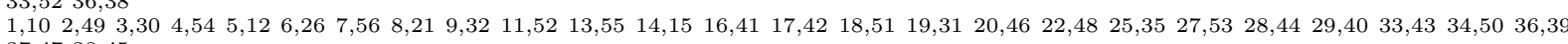
$37,4738,45$

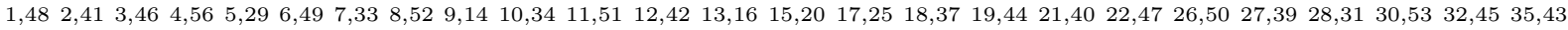
$36,5538,54$

$\begin{array}{lllllllllllllllllllllllllllll}1,55 & 2,31 & 3,54 & 4,23 & 5,18 & 6,15 & 7,19 & 8,51 & 9,30 & 10,38 & 11,17 & 12,48 & 13,22 & 14,40 & 16,53 & 20,49 & 21,56 & 24,47 & 27,50 & 28,43 & 29,39 & 32,41 & 33,46 & 34,44 & 35,45\end{array}$ $36,4237,52$

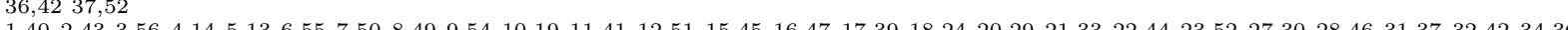
$1,402,43 \quad 3,56$

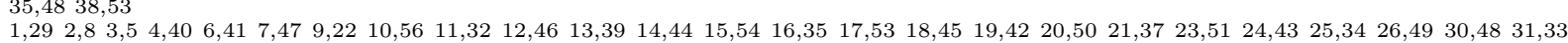
$36,5238,55$

$\begin{array}{llllllllllllllllllllllllllll}1,22 & 2,56 & 3,10 & 4,25 & 5,52 & 6,48 & 7,46 & 8,39 & 9,45 & 11,55 & 12,15 & 13,51 & 14,23 & 16,49 & 17,40 & 18,36 & 19,29 & 20,26 & 21,44 & 24,50 & 30,33 & 31,42 & 32,53 & 34,54 & 35,41\end{array}$ $37,4338,47$

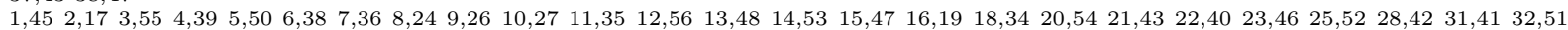
$33,4437,49$

$\begin{array}{lllllllllllllllllllllllllllll}1,36 & 2,39 & 3,34 & 4,51 & 5,44 & 6,46 & 7,23 & 8,40 & 9,47 & 10,48 & 11,18 & 12,52 & 13,43 & 14,56 & 15,50 & 16,42 & 17,49 & 19,32 & 20,55 & 21,45 & 22,25 & 24,31 & 26,35 & 27,54 & 28,38\end{array}$ $33,4137,53$

$\begin{array}{llllllllllllllllllllllllllllllllll}1,41 & 2,38 & 3,45 & 4,5 & 6,50 & 7,27 & 8,48 & 9,28 & 10,11 & 12,34 & 13,56 & 14,24 & 15,17 & 16,25 & 18,46 & 19,55 & 20,44 & 21,47 & 22,43 & 23,49 & 26,52 & 29,53 & 30,51 & 33,54 & 35,42\end{array}$ $36,40 \quad 37,39$

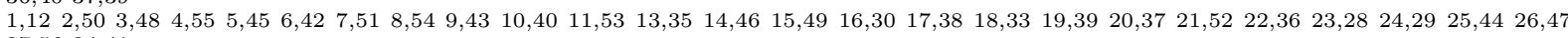
$27,56 \quad 34,41$

$\begin{array}{lllllllllllllllllllllllllllll}1,35 & 2,5 & 3,41 & 4,10 & 6,36 & 7,52 & 8,42 & 9,11 & 12,50 & 13,37 & 14,20 & 15,44 & 16,45 & 17,47 & 18,49 & 19,53 & 21,39 & 22,54 & 23,25 & 24,46 & 26,56 & 27,48 & 28,40 & 29,55 & 30,43\end{array}$

$\begin{array}{llllllllllllllllllllllllllllll}31,51 & 32,38 \\ 1,43 & 2,29 & 3,6 & 4,16 & 5,53 & 7,42 & 8,44 & 9,17 & 10,15 & 11,45 & 12,49 & 13,19 & 14,54 & 18,35 & 20,48 & 21,51 & 22,52 & 23,47 & 24,40 & 25,41 & 26,55 & 27,37 & 28,30 & 31,46 & 32,39\end{array}$ $\begin{array}{lll}1,43 & 2,29 & 3,6\end{array}$

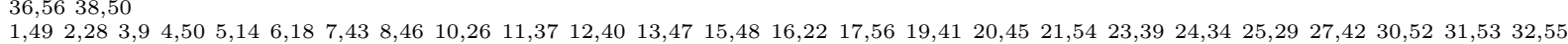
$33,5138,44$

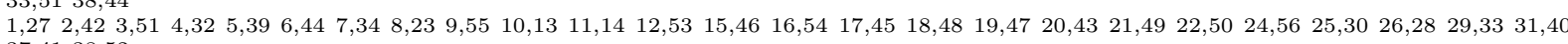
$37,41 \quad 38,52$

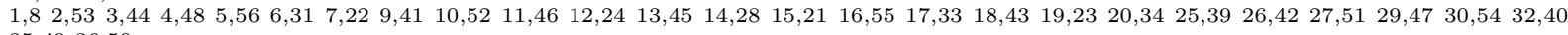
$35,4936,50$

$\begin{array}{llllllllllllllllllllllllll}1,23 & 2,48 & 3,21 & 4,45 & 5,11 & 6,54 & 7,39 & 8,56 & 9,40 & 10,14 & 12,13 & 15,31 & 16,52 & 17,55 & 18,26 & 19,49 & 20,47 & 22,51 & 24,33 & 25,42 & 27,35 & 28,41 & 29,44 & 30,46 & 32,50\end{array}$ $34,5336,43$ 
$\begin{array}{llllllllllllllllllll}1,20 & 2,21 & 3,31 & 4,11 & 5,35 & 6,14 & 7,9 & 8,37 & 10,16 & 12,29 & 13,36 & 15,32 & 17,28 & 18,22 & 19,33 & 23,27 & 24,26 & 25,38 & 30,34\end{array}$ $\begin{array}{lllllllllllllllllllllllll}1,26 & 2,30 & 3,8 & 4,34 & 5,19 & 6,12 & 7,38 & 9,37 & 10,33 & 11,28 & 13,23 & 14,36 & 15,18 & 16,29 & 17,27 & 20,31 & 21,25 & 22,35 & 24,32\end{array}$ $\begin{array}{llllllllllllllllllllll}1,3 & 2,36 & 4,29 & 5,26 & 6,23 & 7,21 & 8,33 & 9,16 & 10,37 & 11,30 & 12,35 & 13,17 & 14,31 & 15,25 & 18,28 & 19,38 & 20,24 & 22,34 & 27,32\end{array}$ $\begin{array}{llllllllllllllllllllllll}1,9 & 2,4 & 3,14 & 5,36 & 6,27 & 7,10 & 8,38 & 11,31 & 12,20 & 13,25 & 15,23 & 16,34 & 17,24 & 18,21 & 19,37 & 22,33 & 26,32 & 28,29 & 30,35\end{array}$ $\begin{array}{llllllllllllllllllll}1,38 & 2,15 & 3,29 & 4,6 & 5,21 & 7,28 & 8,26 & 9,36 & 10,31 & 11,19 & 12,18 & 13,32 & 14,25 & 16,33 & 17,30 & 20,22 & 23,37 & 24,27 & 34,35\end{array}$

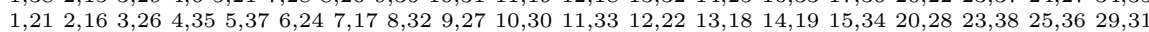

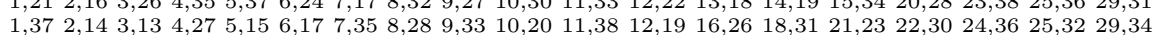
$\begin{array}{lllllllllllllllllll}1,37 & 2,14 & 3,13 & 4,27 & 5,15 & 6,17 & 7,35 & 8,28 & 9,33 & 10,20 & 11,38 & 12,19 & 16,26 & 18,31 & 21,23 & 22,30 & 24,36 & 25,32 & 29,34 \\ 1,7 & 2,3 & 4,26 & 5,17 & 6,11 & 8,25 & 9,23 & 10,28 & 12,32 & 13,33 & 14,18 & 15,35 & 16,38 & 19,34 & 20,21 & 22,24 & 27,31 & 29,37 & 30,36\end{array}$ $\begin{array}{llllllllllllllllllllllll}1,34 & 2,37 & 3,20 & 4,18 & 5,31 & 6,19 & 7,26 & 8,30 & 9,21 & 10,36 & 11,25 & 12,17 & 13,29 & 14,33 & 15,22 & 16,23 & 24,35 & 27,38 & 28,32\end{array}$ $\begin{array}{lllllllllllllllllllllllll}1,16 & 2,9 & 3,15 & 4,28 & 5,10 & 6,8 & 7,32 & 11,34 & 12,23 & 13,27 & 14,35 & 17,20 & 18,30 & 19,24 & 21,26 & 22,37 & 25,31 & 29,36 & 33,38\end{array}$ $\begin{array}{lllllllllllllllllllllll}1,4 & 2,25 & 3,11 & 5,9 & 6,10 & 7,31 & 8,34 & 12,30 & 13,24 & 14,26 & 15,28 & 16,20 & 17,19 & 18,23 & 21,29 & 22,38 & 27,36 & 32,35 & 33,37\end{array}$ $\begin{array}{llllllllllllllllllllllll}1,17 & 2,27 & 3,28 & 4,19 & 5,7 & 6,34 & 8,22 & 9,13 & 10,12 & 11,23 & 14,32 & 15,36 & 16,31 & 18,38 & 20,30 & 21,24 & 25,37 & 26,33 & 29,35\end{array}$ $\begin{array}{llllllllllllllllllllllll}1,28 & 2,34 & 3,38 & 4,31 & 5,24 & 6,20 & 7,37 & 8,17 & 9,25 & 10,29 & 11,13 & 12,26 & 14,16 & 15,27 & 18,32 & 19,30 & 21,36 & 22,23 & 33,35\end{array}$

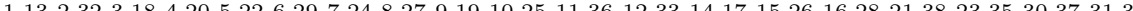

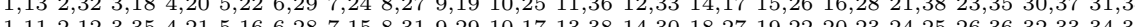

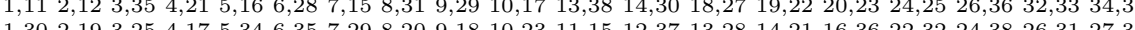
$\begin{array}{llllllllllllllllllllllllll}1,30 & 2,19 & 3,25 & 4,17 & 5,34 & 6,35 & 7,29 & 8,20 & 9,18 & 10,23 & 11,15 & 12,37 & 13,28 & 14,21 & 16,36 & 22,32 & 24,38 & 26,31 & 27,33\end{array}$ $\begin{array}{llllllllllllllllllllllllllllllll}1,18 & 2,35 & 3,19 & 4,38 & 5,27 & 6,30 & 7,14 & 8,16 & 9,24 & 10,21 & 11,20 & 12,25 & 13,31 & 15,33 & 17,32 & 22,29 & 23,34 & 26,37 & 28,36\end{array}$ $\begin{array}{lllllllllllllllllllllll}1,5 & 2,11 & 3,36 & 4,24 & 6,21 & 7,12 & 8,35 & 9,20 & 10,32 & 13,15 & 14,27 & 16,37 & 17,34 & 18,29 & 19,25 & 22,26 & 23,31 & 28,33 & 30,38\end{array}$ 